\title{
IS PROTOSTELLAR HEATING SUFFICIENT TO HALT FRAGMENTATION? A CASE STUDY OF THE MASSIVE PROTOCLUSTER G8.68-0.37
}

\author{
S. N. Longmore ${ }^{1}$, T. Pillai ${ }^{2}$, E. Keto ${ }^{3}$, Q. Zhang ${ }^{3}$, And K. Qiu ${ }^{4}$ \\ ${ }^{1}$ European Southern Observatory, Karl-Schwarzschild-Str. 2, 85748 Garching bei München, Germany \\ ${ }^{2}$ Caltech Astronomy Department, 1200 East California Blvd., Pasadena, CA 91125, USA \\ ${ }^{3}$ Harvard-Smithsonian Center for Astrophysics, 60 Garden Street, Cambridge, MA 02138, USA; slongmore@cfa.harvard.edu \\ ${ }^{4}$ Max-Planck-Institut für Radioastronomie, Auf dem Hügel 69, 53121 Bonn, Germany \\ Received 2010 July 20; accepted 2010 November 3; published 2010 December 20
}

\begin{abstract}
If star formation proceeds by thermal fragmentation and the subsequent gravitational collapse of the individual fragments, how is it possible to form fragments massive enough for $\mathrm{O}$ and $\mathrm{B}$ stars in a typical star-forming molecular cloud where the Jeans mass is about $1 M_{\odot}$ at the typical densities $\left(10^{4} \mathrm{~cm}^{-3}\right)$ and temperatures $(10 \mathrm{~K})$ ? We test the hypothesis that a first generation of low-mass stars may heat the gas enough that subsequent thermal fragmentation results in fragments $\geqslant 10 M_{\odot}$, sufficient to form B stars. We combine ATCA and Submillimeter Array observations of the massive star-forming region G8.68-0.37 with radiative transfer modeling to derive the present-day conditions in the region and use this to infer the conditions in the past, at the time of core formation. Assuming that the current mass/separation of the observed cores equals the fragmentation Jeans mass/length and the region's average density has not changed requires the gas temperature to have been $100 \mathrm{~K}$ at the time of fragmentation. The postulated first generation of low-mass stars would still be around today, but the number required to heat the cloud exceeds the limits imposed by the observations. Several lines of evidence suggest the observed cores in the region should eventually form $\mathrm{O}$ stars yet none have sufficient raw material. Even if feedback may have suppressed fragmentation, it was not sufficient to halt it to this extent. To develop into $\mathrm{O}$ stars, the cores must obtain additional mass from outside their observationally defined boundaries. The observations suggest that they are currently fed via infall from the very massive reservoir $\left(\sim 1500 M_{\odot}\right)$ of gas in the larger parsec scale cloud around the star-forming cores. This suggests that massive stars do not form in the collapse of individual massive fragments, but rather in smaller fragments that themselves continue to gain mass by accretion from larger scales.
\end{abstract}

Key words: ISM: clouds - ISM: molecules - stars: early-type - stars: formation - stars: pre-main sequence

Online-only material: color figures

\section{INTRODUCTION}

Massive stars play an influential role in shaping the universe. Observations show that the substantial majority form in clusters (Lada \& Lada 2003) yet the physical processes governing the fragmentation and collapse of their natal molecular cloud, a crucial step in determining important parameters such as the number of massive stars and their final stellar mass, remains an unsolved problem. Infrared dark clouds, thought to be examples of the birth sites of massive clusters, are observed to have temperatures of 10-20 K and contain many hundreds to thousands of solar masses of gas (Pillai et al. 2006; Ragan et al. 2006; Rathborne et al. 2006, 2010; Swift 2009; Zhang et al. 2009). It remains an open question whether these initial conditions can produce fragments large enough to form high-mass stars through direct collapse, without the cores themselves sub-fragmenting first. Radiative feedback (heating) from embedded, low-mass protocluster members has been proposed as a mechanism to delay fragmentation by changing the effective equation of state (Krumholz et al. 2007). In this paper, we consider a more direct mechanism to suppress the sub-fragmentation. Through observations of a very young high-mass protocluster, we investigate whether heating from a first generation of low-mass stars can raise the cloud temperature enough that subsequent thermal fragmentation can produce fragments with sufficient mass to form high-mass stars.

\subsection{The IRAS 18032-2137 Complex}

Figure 1 shows the IRAS 18032-2137 star-forming complex, comprised of three distinct regions separated by a few arcminutes on the sky. The most evolved of these is the stellar cluster, BDS2003-3 $\left(\alpha_{\mathrm{J} 2000}=18: 06: 15, \delta_{\mathrm{J} 2000}=-21: 37: 30\right.$; Bica et al. 2003). The high visual extinction and association with both near-IR nebulosity (Longmore \& Burton 2009) and radio continuum emission (G8.662-0.342; Becker et al. 1994) make this most likely a massive, heavily embedded cluster. One arcminute east of the cluster lies the ultra-compact H II region G8.67-0.36 (Wood \& Churchwell 1989), coincident with a compact submillimeter continuum peak (Hill et al. 2005, 2006; Thompson et al. 2006). One arcminute (projected separation of $1.4 \mathrm{pc}$ ) northeast along the same submillimeter continuum filament lies a second submillimeter continuum peak, G8.68-0.37. Both G8.67-0.36 and G8.68-0.37 are associated with $\mathrm{H}_{2} \mathrm{O}$, class II $\mathrm{CH}_{3} \mathrm{OH}$, and $\mathrm{OH}$ maser emission (Hofner \& Churchwell 1996; Walsh et al. 1998; Forster \& Caswell 1989; Caswell 1998; Val'tts et al. 2000). Hill et al. (2010) show that G8.68-0.37 is massive $\left(\sim 1.5 \times 10^{3} M_{\odot}\right)$ and has a high luminosity $\left(\sim 10^{4} L_{\odot}\right)$ yet deep centimeter continuum observations reveal no compact (M. Pestallozi 2010, private communication) or extended (Longmore et al. 2009) free-free emission. At a distance of $4.8 \mathrm{kpc}$ (Purcell et al. 2006), the $3 \sigma$ upper limit of $1.7 \mathrm{mJy}$ at $3.6 \mathrm{~cm}$ (M. Pestallozi 2010, private 


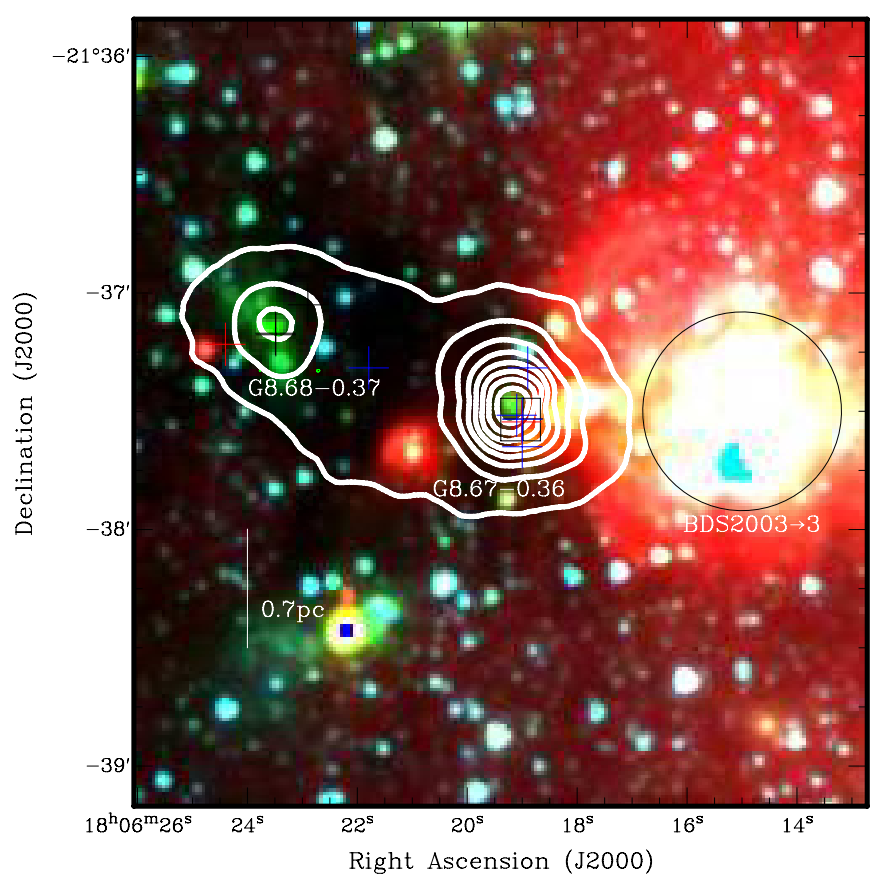

Figure 1. IRAS 18032-2137 star-forming complex. A GLIMPSE three-color image at $3.6 \mu \mathrm{m}, 4.5 \mu \mathrm{m}$, and $8.0 \mu \mathrm{m}$ is overlaid with SCUBA $850 \mu \mathrm{m}$ continuum emission contours (Hill et al. 2006). The complex comprises three distinct regions: (1) the stellar cluster, BDS2003-3 (Bica et al. 2003), with the approximate extent reported by Bica et al. (2003) indicated by the circle; (2) the ultra-compact H II region G8.67-0.36 (Wood \& Churchwell 1989; centimeter continuum position illustrated by box); and (3) a massive star-forming core prior to formation of an $\mathrm{H}$ II region, G8.68-0.37. $\mathrm{H}_{2} \mathrm{O}, \mathrm{CH}_{3} \mathrm{OH}$, and $\mathrm{OH}$ maser emission (Hofner \& Churchwell 1996; Walsh et al. 1998; Forster \& Caswell 1989; Caswell 1998; Val'tts et al. 2000) are shown as blue, black, and red crosses, respectively. The line toward the lower left gives the physical scale for the source distance of $4.8 \mathrm{kpc}$ (Purcell et al. 2006).

communication) corresponds to a Lyman-continuum photon rate of $1.65 \times 10^{46} \mathrm{~s}^{-1}$, implying no stars earlier than B0-B1 are present in the cluster. All of this points to the nature of G8.68-0.37 as a very young massive star-forming region, prior to the formation of ultra-compact or hyper-compact $\mathrm{H}$ II regions.

$\mathrm{NH}_{3}$ observations of G8.68-0.37 show (1) an extended cold component seen in the $\mathrm{NH}_{3}(1,1)$ and $(2,2)$ transitions with a morphology similar to the submillimeter continuum emission and (2) a warmer component in $\mathrm{NH}_{3}(4,4)$ and $(5,5)$, unresolved at $8^{\prime \prime}$ at the peak of the submillimeter continuum and methanol maser emission (Pillai et al. 2007; Longmore et al. 2007). This suggests that G8.68-0.37 is internally heated by young (proto)stars of at least several $M_{\odot}$. Strong infall profiles are seen in the $3 \mathrm{~mm}$ Mopra spectra of $\mathrm{HCO}^{+}, \mathrm{HNC}$, and even ${ }^{13} \mathrm{CO}$ (Purcell et al. 2006, 2009), while Harju et al. (1998) report strong $\mathrm{SiO}$ emission indicative of shocks caused by outflows - further evidence that star formation is already underway in the protocluster.

In summary, G8.68-0.37 is a relatively isolated massive star-forming core with a symmetric and centrally peaked dust profile. It is still in the earliest stages of forming a massive protocluster and has already begun to significantly heat the gas in the protocluster center. As such it appears a good candidate region for testing whether energetic feedback can act sufficiently quickly to suppress thermal fragmentation.
Table 1

Overview of the Observing Setups

\begin{tabular}{lccc}
\hline \hline $\begin{array}{c}\text { Array } \\
\text { Configuration }\end{array}$ & $\begin{array}{c}\text { Frequency } \\
(\mathrm{GHz})\end{array}$ & $\begin{array}{c}\text { Cont. rms } \\
\left(\mathrm{mJy} \mathrm{beam}^{-1}\right)\end{array}$ & $\begin{array}{c}\text { Beam } \\
\left({ }^{\prime \prime}\right)\end{array}$ \\
\hline Sub-compact & 279 & 7.3 & $7.4 \times 6.2$ \\
Compact & 230 & 1.4 & $3.1 \times 2.0$ \\
Extended & 217 & 3.4 & $1.1 \times 1.0$
\end{tabular}

Notes. The first two columns show the array configuration and observing frequencies. The sub-compact, compact, and extended array configurations have baselines ranging from 31-89 m, 61-192 m, and 82-247 m, respectively. The third and final columns give the continuum sensitivity and synthesized beam size.

\section{OBSERVATIONS AND DATA REDUCTION}

The data were taken with the Submillimeter Array ${ }^{5}$ (SMA) between 2007 September 2 and 2008 September 17 in three individual tracks between 217 and $279 \mathrm{GHz}$ in the sub-compact, compact, and extended array configurations. At these frequencies, the SMA primary beam (field of view) is $\sim 45^{\prime \prime}-58^{\prime \prime}$, sufficient to cover the extent of the single-dish continuum emission (see Figure 1) in a single pointing centered at $\alpha_{J 2000}=$ 18:06:23.47, $\delta_{J 2000}=-21: 37: 7.6$. For each track, this sky position was observed for periods of 10-15 minutes on-source, interspersed with observations of two bright, nearby calibrators (1733-130 and 1911-201). Two bandpass calibrators, 3c279 and $3 \mathrm{c} 454.3$, were observed for approximately an hour at the start and end of each observation. At least one primary flux calibrator (Uranus, Neptune, and Titan) was observed for each track. The absolute flux scale is estimated to be accurate to $\sim 15 \%$. In all cases, the weather was very stable and the resulting amplitude/phase stability was good. The data were calibrated using the MIR IDL package ${ }^{6}$ and exported to Miriad to be imaged and cleaned. A zeroth-order polynomial was fitted to the line-free channels and subtracted from the visibilities to separate the line and continuum emission, which were imaged separately. Table 1 lists the observing setup, continuum sensitivity, and resolution for each of the tracks. The results are shown in Section 3.1.

\section{RESULTS}

\subsection{SMA Data}

In the sub-compact and compact-configuration data, the continuum morphology is similar to that seen in the single-dish observations - a single component, centrally peaked toward the methanol maser position. However, as shown in Figure 2, the SMA extended configuration observations resolve the emission into three components, named MM1 to MM3 in order of peak intensity. These are separated by a minimum of $1^{\prime \prime} .2$ and a maximum of 2 .' 2 , corresponding to projected distances of $\sim 6200 \mathrm{AU}$ and $\sim 9700 \mathrm{AU}$, respectively. Table 2 gives the properties of the millimeter continuum detections.

Figures 3 and 4 show the $230 \mathrm{GHz}$ lower and upper sideband spectra, respectively, toward the peak of the continuum emission from the compact-configuration data. In addition to the ${ }^{13} \mathrm{CO}(2 \rightarrow 1)$ and ${ }^{12} \mathrm{CO}(2 \rightarrow 1)$ emission, the spectra show

5 The Submillimeter Array is a joint project between the Smithsonian Astrophysical Observatory and the Academia Sinica Institute of Astronomy and Astrophysics and is funded by the Smithsonian Institution and the Academia Sinica (Ho et al. 2004).

6 http://www.cfa.harvard.edu/ cqi/mircook.html 


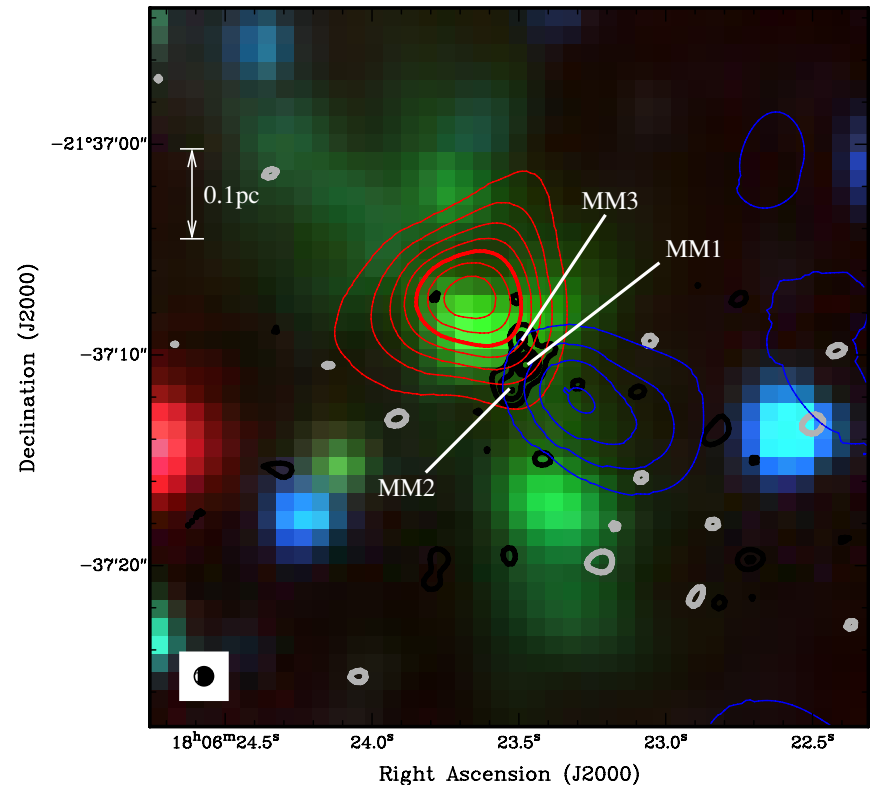

Figure 2. Three-color GLIMPSE image at 3.6, 4.5, and $8.0 \mu \mathrm{m}$ overlaid with (1) the $217 \mathrm{GHz}$ SMA extended configuration continuum image (gray $(-3 \sigma)$ and black $(3 \sigma, 6 \sigma, 9 \sigma, \ldots)$ contours: $\sigma=2.6 \mathrm{mJy})$ and $(2)$ the ${ }^{12} \mathrm{CO}(2 \rightarrow 1)$ emission integrated from 29.3 to $36.3 \mathrm{~km} \mathrm{~s}^{-1}$ (blue contours) and 45 to $74 \mathrm{~km} \mathrm{~s}^{-1}$ (red contours). The blue and red lobes of the molecular outflow lie along a similar axis as the extended $4.5 \mu \mathrm{m}$ emission-a well-known tracer of gas shocked in outflows. The three SMA $1 \mathrm{~mm}$ emission peaks are labeled MM1 to MM3 in order of peak $1 \mathrm{~mm}$ continuum emission flux. The brightest component, MM1, is found at the center of the outflow lobes and appears the best candidate for the outflow driving source. The filled black circle in the lower left corner gives the synthesized beam of the SMA extended configuration observations. The bar in the upper left corner illustrates the physical scale for the source distance of $4.8 \mathrm{kpc}$.

Table 2

Properties of the millimeter Continuum Sources Detected with the SMA in the Extended Array Configuration

\begin{tabular}{lcccc}
\hline \hline Source & Flux (mJy) & Flux Peak (mJy) & R.A. & Decl. \\
\hline MM1 & 45 & 37 & $18: 06: 23.479$ & $-21: 37: 10.5$ \\
MM2 & 30 & 24 & $18: 06: 23.522$ & $-21: 37: 11.7$ \\
MM3 & 17 & 14 & $18: 06: 23.494$ & $-21: 37: 09.1$ \\
\hline
\end{tabular}

several more complex molecules (e.g., $\mathrm{CH}_{3} \mathrm{OH}$ and $\mathrm{CH}_{3} \mathrm{CN}$ ) confirming that the temperature is sufficiently high for these molecules to have evaporated off the dust grains and into the gas phase. However, the spectra do not display the rich inventory of molecular lines seen toward hot molecular cores (see Cesaroni 2005 for a review) suggesting this region is an intermediate evolutionary stage between the cold and hot core stages. A similar result is found for the extended configuration observations which were tuned to $217 \mathrm{GHz}$ (instead of $230 \mathrm{GHz}$ ). Figure 5 shows the ${ }^{12} \mathrm{CO}(2 \rightarrow 1)$ channel maps in which a bipolar morphology is clearly seen at opposite sides of the systemic $V_{\mathrm{LSR}}$ $\left(37.2 \mathrm{~km} \mathrm{~s}^{-1}\right)$, indicative of a molecular outflow. The properties of this outflow are discussed in more detail in Section 4.2.

The emission from more complex species detected in the $230 \mathrm{GHz}$ compact configuration is generally unresolved within the $3^{\prime \prime} \times 2^{\prime \prime}$ beam and encompasses MM1 to MM3. This emission is also coincident with the $\mathrm{NH}_{3}(4,4)$ and $(5,5)$ and methanol maser emission. The spectral lines are typically well fit as a single Gaussian at the $V_{\mathrm{LSR}}$, with linewidth $\sim 5 \mathrm{~km} \mathrm{~s}^{-1}$ and no sign of any coherent, large-scale velocity structure.

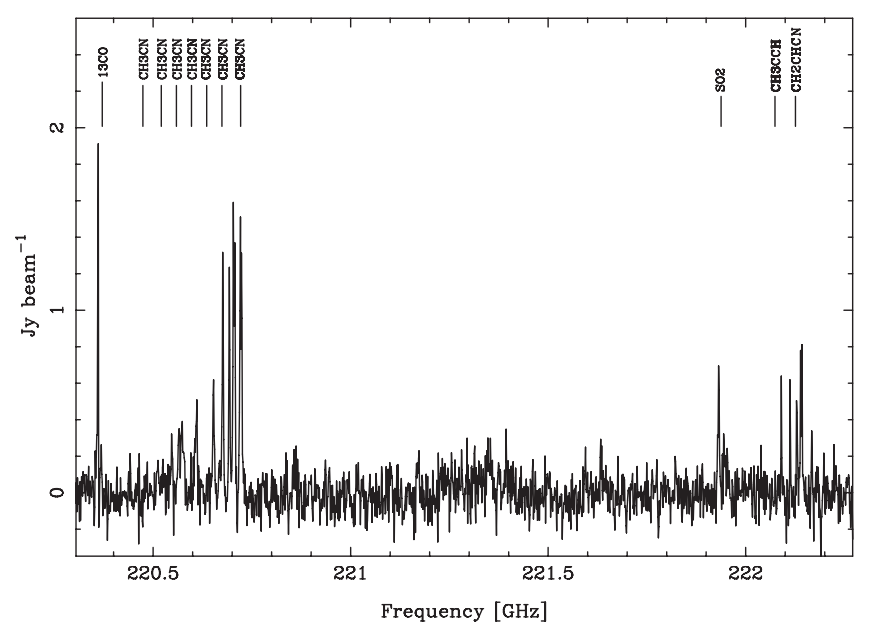

Figure 3. Lower sideband spectrum of the $230 \mathrm{GHz}$, compact-configuration SMA data taken at the position of peak continuum emission and smoothed to a resolution of $1.75 \mathrm{~km} \mathrm{~s}^{-1}$. Transitions with two consecutive channels $>3 \sigma\left(\sigma=92 \mathrm{mJy} \mathrm{channel}^{-1}\right)$ are labeled above the spectrum at frequencies (corrected for the source $V_{\mathrm{LSR}}$ of $37.2 \mathrm{~km} \mathrm{~s}^{-1}$ ) taken from the NIST catalog (Lovas 2004). In addition to the ${ }^{13} \mathrm{CO}(2 \rightarrow 1)$ and ${ }^{12} \mathrm{CO}(2 \rightarrow 1)$ emission, the spectra show several more complex molecules (e.g., $\mathrm{CH}_{3} \mathrm{OH}$ and $\mathrm{CH}_{3} \mathrm{CN}$ ) confirming that the source is warm enough for these molecules to have evaporated off the dust grains and into the gas phase. However, the spectra do not display the rich inventory seen toward hot molecular cores, suggesting that this region is an intermediate evolutionary stage between the cold and hot core stages.

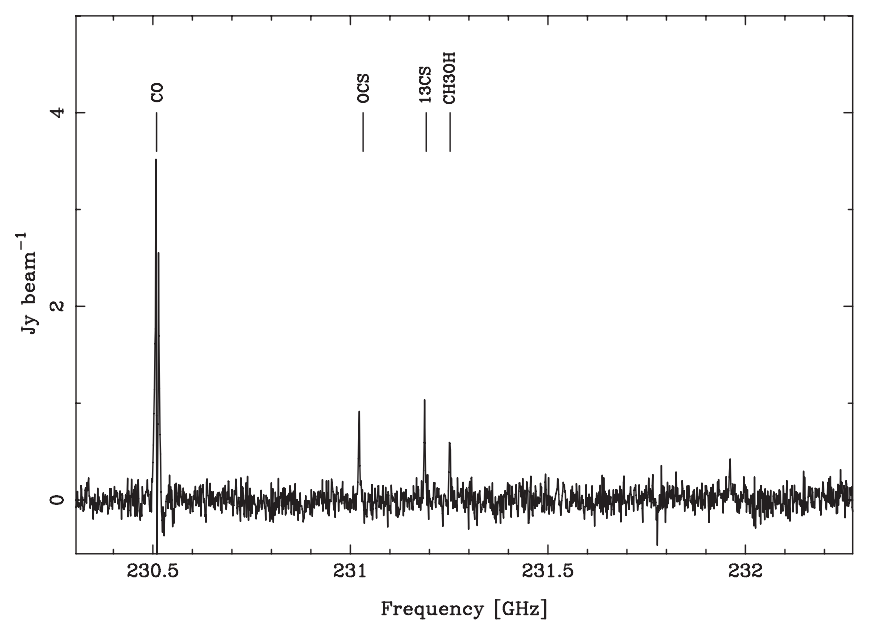

Figure 4. Upper sideband spectrum of the $230 \mathrm{GHz}$, compact-configuration SMA data taken at the position of peak continuum emission and smoothed to a resolution of $1.75 \mathrm{~km} \mathrm{~s}^{-1}$. Transitions with two consecutive channels $>3 \sigma\left(\sigma=94 \mathrm{mJy}_{\text {channel }}{ }^{-1}\right)$ are labeled above the spectrum at frequencies (corrected for the source $V_{\mathrm{LSR}}$ of $37.2 \mathrm{~km} \mathrm{~s}^{-1}$ ) taken from the NIST catalog (Lovas 2004). In addition to the ${ }^{13} \mathrm{CO}(2 \rightarrow 1)$ and ${ }^{12} \mathrm{CO}(2 \rightarrow 1)$ emission, the spectra show several more complex molecules (e.g., $\mathrm{CH}_{3} \mathrm{OH}$ and $\mathrm{CH}_{3} \mathrm{CN}$ ) confirming that the source is warm enough for these molecules to have evaporated off the dust grains and into the gas phase. However, the spectra do not display the rich inventory seen toward hot molecular cores, suggesting that this region is an intermediate evolutionary stage between the cold and hot core stages.

\subsection{Spitzer Data}

Figure 2 shows a three-color GLIMPSE image (Benjamin et al. 2003) at 3.6, 4.5, and $8.0 \mu \mathrm{m}$ toward the region taken with IRAC on the Spitzer Space Telescope. There is a clear bipolar nebulosity in the image, prominent in all four IRAC bands. The black contours show the positions of the three millimeter continuum cores detected with the SMA relative to the IRAC emission. Of the three millimeter cores, the nebulosity is most 


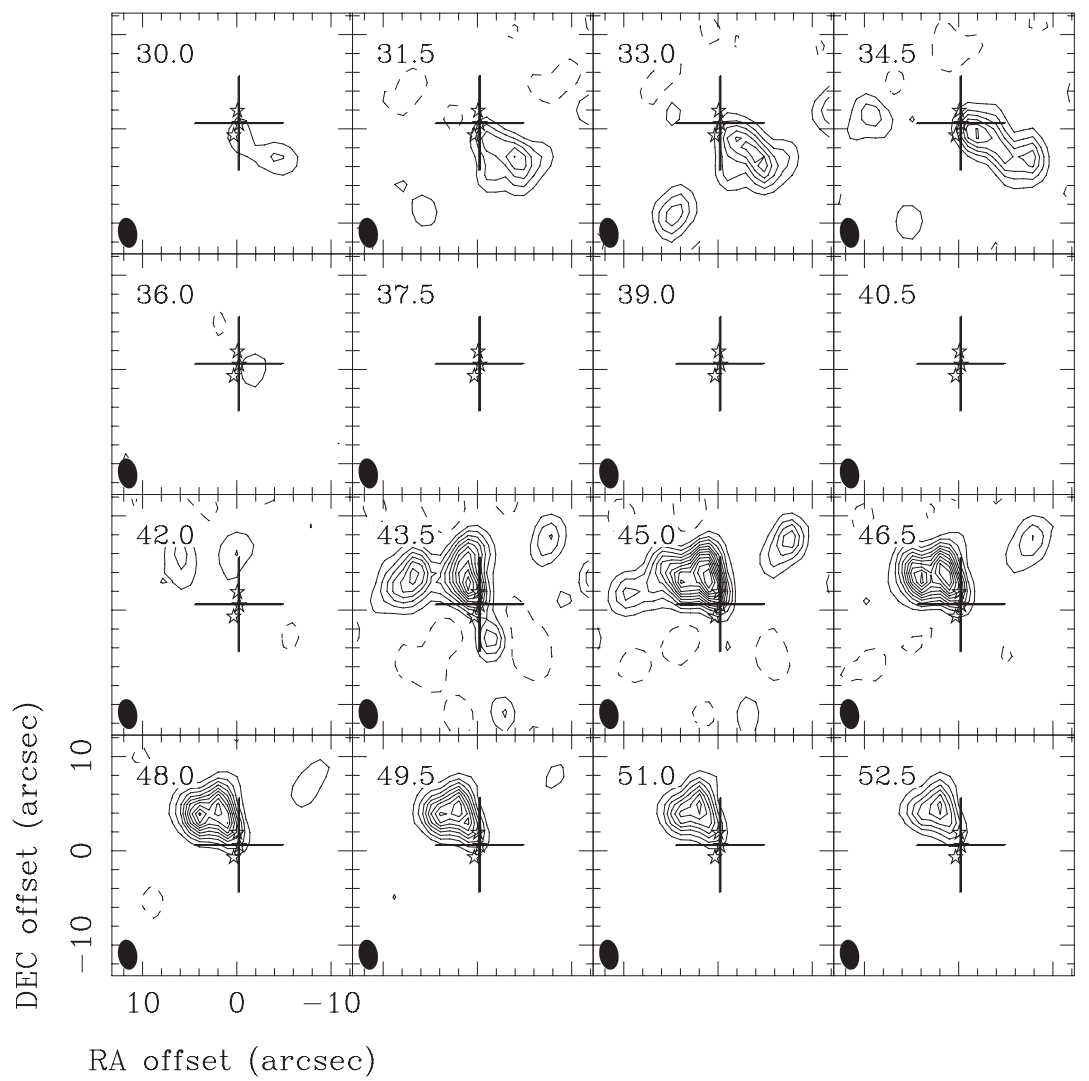

Figure 5. ${ }^{12} \mathrm{CO}(2 \rightarrow 1)$ channel map toward G8.68-0.37. The $V_{\mathrm{LSR}}\left(\right.$ in $\mathrm{km} \mathrm{s}^{-1}$ ) of each frame is shown on the top left of each panel and the synthesized beam size is shown as a filled ellipse in the lower left corner of each frame. The center of the coordinate system and location of the cross show the methanol maser position at $\alpha_{J 2000}=18: 06: 23.47, \delta_{J 2000}=-21: 37: 10.6$. The contours start at $\pm 5 \sigma$ and increment in $5 \sigma$ intervals $\left(\sigma=140 \mathrm{mJy}\right.$ beam $\left.{ }^{-1}\right)$. For reference, the three stars show the location of MM1, MM2, and MM3.

closely associated with MM1. In the longer wavelengths (24 and $70 \mu \mathrm{m})$ and lower resolution $\left(7.5^{\prime \prime}-22^{\prime \prime}\right)$ MIPSGAL data (Carey et al. 2009), only a single, bright (1 Jy integrated flux at $24 \mu \mathrm{m})$ emission peak is seen with MIPS.

\section{ANALYSIS}

\subsection{Total Luminosity of $G 8.68-0.37$}

While luminosity estimates for this region are available from the literature, access to recent Spitzer and deep near-IR data not available to previous authors enables us to provide tighter constraints. We used the Spitzer 24 and $70 \mu \mathrm{m}$, SCUBA 450 and $850 \mu \mathrm{m}$, and SIMBA $1.3 \mathrm{~mm}$ (Hill et al. 2005) data to estimate the total luminosity of the system. Deep, near-IR imaging shows that this region is infrared dark down to 18th magnitude at 2.2 $\mu \mathrm{m}$ (Longmore \& Burton 2009), giving confidence that most of the luminosity is emitted at the longer wavelengths we used to construct the spectral energy distribution. Based on the similar size and morphology of the emission from $24 \mu \mathrm{m}$ to $1.3 \mathrm{~mm}$, we used a circular aperture of radius $15^{\prime \prime}$ to derive the total flux at each wavelength. We fit the resulting spectral energy distribution as a graybody and, integrating under the resultant fit, estimate the protocluster luminosity to be $\sim 1.9 \times 10^{4} L_{\odot}$. The SMA data were not used in the luminosity estimate as comparison with the SIMBA data (see Section 4.3.1) revealed that a large fraction of the total flux has been spatially filtered by the interferometer.

\subsection{Outflow Properties}

As shown in Figure 2, both the $230 \mathrm{GHz}$ compact configuration CO SMA data and Spitzer data reveal a bipolar outflow in an approximately NE-SW orientation. The higher angular resolution, extended configuration, $217 \mathrm{GHz}$ continuum SMA data show core MM1 lies directly along the projected outflow axis of both the $\mathrm{CO}$ and IRAC nebulosity making it most likely to be driving the outflow. Figure 5 shows channel maps of the ${ }^{12} \mathrm{CO}(2 \rightarrow 1)$ emission. Extended emission from the ambient cloud is filtered out by the interferometer (our shortest baseline corresponds to a spatial filtering of emission more extended than $30^{\prime \prime}$ ) close to the local standard of rest (LSR) velocity of $37.2 \mathrm{~km} \mathrm{~s}^{-1}$ (Purcell et al. 2006, 2009). To determine the outflow properties, we first separated the outflow into its two component lobes using a $3 \sigma$ cutoff in the ${ }^{12} \mathrm{CO}$ data cube at velocities 29.3-36.3 $\mathrm{km} \mathrm{s}^{-1}$ (blue) and 45-74 $\mathrm{km} \mathrm{s}^{-1}$ (red). Velocities closer to the LSR velocity were avoided to prevent contamination from the ambient low density gas.

The outflow mass was derived following Scoville et al. (1986), assuming a $\mathrm{CO}$ excitation temperature of $30 \mathrm{~K}$. We defined a blue and red polygon covering the spatial extent of the outflow emission shown in Figure 2 and derived an average spectrum for both ${ }^{12} \mathrm{CO}$ and ${ }^{13} \mathrm{CO}$. Where ${ }^{13} \mathrm{CO}$ was detected, we estimated the ${ }^{12} \mathrm{CO}$ optical depth by assuming the ${ }^{13} \mathrm{CO}$ emission to be optically thin with an abundance ratio to ${ }^{12} \mathrm{CO}$ of 89 (see Wilson \& Matteucci 1992). The ${ }^{12} \mathrm{CO}$ optical depth was then used to compute the $\mathrm{CO}$ column density. For velocities with no ${ }^{13} \mathrm{CO}$ emission, optically thin ${ }^{12} \mathrm{CO}$ emission was assumed. We thus derive a total outflow mass of $\sim 6 M_{\odot}$.

Based on the distance between the edge of the lobes through the projected center, the outflow has a dynamical time of $\sim 1.3 \times 10^{4}$ yr. The derived outflow rate is therefore $\sim 4.4 \times$ $10^{-4} M_{\odot} \mathrm{yr}^{-1}$. We emphasize that due to missing flux (see 


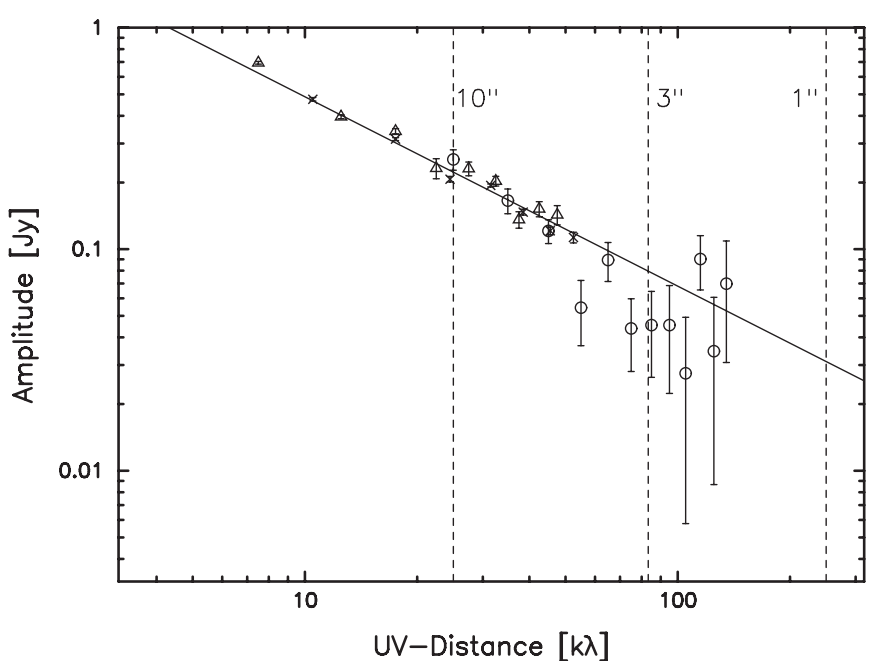

Figure 6. UV-distance vs. visibility amplitude for the $1 \mathrm{~mm}$ continuum emission observed with the SMA. Visibilities from the compact, sub-compact, and extended array configurations are shown as triangles, squares, and circles, respectively. The error bars show the uncertainty calculated from the variation in amplitude for the visibilities binned for each point. The solid line shows the weighted least-squares fit to the data-assuming $T \propto r^{-0.33}$ as expected for an internally heated source (see Section 4.3.1), the best-fit density profile is given by $\rho \propto r^{-1.8 \pm 0.2}$. The dashed lines show the corresponding angular scale at $10^{\prime \prime}$ (left), $3^{\prime \prime}$ (center), and $1^{\prime \prime}$ (right). While the assumption of spherical symmetry appears a good fit to the data at large spatial scales, it is clear that this assumption breaks down at $\lesssim 3^{\prime \prime}$, where the extended configuration observations resolve MM1, MM2, and MM3.

Figure 5) and optical depth effects, the mass and outflow rate could be underestimated. Still, for the luminosity of the protocluster, the outflow properties are in good agreement with those seen toward other high-mass star-forming regions (see, for example, Shepherd \& Churchwell 1996; Beuther et al. 2002; Zhang et al. 2001, 2005; Qiu et al. 2009).

Outflow studies in high-mass star-forming regions are often hampered by the complexity of the observed emission. In most cases, one sees multiple outflows, many with unknown driving sources, which may be difficult to disentangle. While there are potentially signs of additional outflows in Figure 5 (e.g., the southeast clump at offset $5^{\prime \prime},-10^{\prime \prime}$, and 31.5 to $34.5 \mathrm{~km} \mathrm{~s}^{-1}$ and the northwest clump at offset $8^{\prime \prime}, 8^{\prime \prime}$, and 43.5 to $48.0 \mathrm{~km} \mathrm{~s}^{-1}$ may make another outflow), G8.68-0.37 is dominated by the simple, bipolar morphology discussed above which is wide angled, especially the red lobe. As shown in Figure 2, the CO outflow is well aligned with the shocked emission seen in IRAC bands presumably from the interaction of the outflow with the molecular envelope.

Cyganowski et al. (2009) find that methanol masers are detected toward a large fraction of massive star-forming regions associated with extended $4.5 \mu \mathrm{m}$ emission, so the association of the two in G8.68-0.37 is not surprising. However, the resolution of the SMA observations is not sufficient to add to the ongoing debate whether these masers are associated with outflows or disks (see De Buizer et al. 2009 and references therein).

\subsection{Data Modeling}

\subsubsection{Temperature and Density Profile Modeling of Continuum Data}

Following Zhang et al. (2009; see also Takahashi et al. 2009), we used the measured visibility amplitude of the SMA continuum data as a function of $u v$-distance to determine the spatial density profile of G8.68-0.37. Assuming that the density, $\rho$, and dust temperature, $T_{\text {dust }}$, scale as a power law with ra- dius (i.e., $T_{\text {dust }}(r) \propto r^{-\kappa_{T}}$ and $\rho(r) \propto r^{-\kappa_{\rho}}$ ) and the dust is centrally heated, then the flux density from dust emission, $F$, is given by $F \propto \int \rho T_{\text {dust }} d S$, where $S$ is the length along the line of sight. For $\kappa_{\rho}+\kappa_{T}>1$, in the image domain the flux density scales as $F \propto r^{-\left(\kappa_{\rho}+\kappa_{T}-1\right)}$, while in the $u v$ plane this corresponds to $A \propto S_{u v}^{\left(\kappa_{\rho}+\kappa_{T}-3\right)}$, where $A$ is the visibility amplitude and $S_{u v}$ is the $u v$-distance. Figure 6 shows the visibility amplitude as a function of $u v$-distance for the sub-compact (triangles), compact (crosses), and extended (circles) array configurations, scaled by the observing frequency. The error bars show the uncertainty calculated from the variation in amplitude for the visibilities binned for each point. Assuming a temperature exponent $\kappa_{T}=0.33$ (appropriate for the dust being centrally heated), the weighted least-squares fit to the data (shown as a line in Figure 6) reveals $\kappa_{\rho}=1.8 \pm 0.2$. The intercept of the fit, corresponding to the total flux, agrees well with the value of $3.4 \mathrm{Jy}$ reported in Hill et al. (2005).

\subsubsection{Radiative Transfer Modeling of $\mathrm{NH}_{3}$ Data}

To determine a second, independent measurement of the spatial temperature and density profile, we used radiative transfer modeling to fit the Longmore et al. (2007) $\mathrm{NH}_{3}(1,1),(4,4)$, and $(5,5)$ data. The radiative transfer code, MOLLIE, ${ }^{7}$ can deal with arbitrary three-dimensional geometries, but based on (1) the morphology of the single-dish submillimeter continuum emission and (2) analysis of the density profile in Section 4.3.1, a spherically symmetric model was chosen as a good approximation, at least down to spatial scales of $\sim 0.05$ pc probed by the extended configuration SMA observations. A model radius of $0.58 \mathrm{pc}$ was chosen based on the extent of the single-dish submillimeter continuum emission. The temperature, $T_{K}$, and density, $\rho$, were again parameterized with radius, $r$, as power laws: $\rho(r)=\rho_{1 / 2} r^{-\kappa_{\rho}}$ and $T_{K}(r)=T_{1 / 2} r^{-\kappa_{T}}$, where $\rho_{1 / 2}$ and $T_{1 / 2}$ are the density and temperature at the half-radius, $0.29 \mathrm{pc}$. An additional constant velocity component, $\Delta V_{\mathrm{NT}}$, was included to model non-thermal support (e.g., from turbulence), resulting in a five-parameter model for the cloud. The $\mathrm{NH}_{3}$ to $\mathrm{H}_{2}$ abundance was fixed at $5 \times 10^{-9}$ (derived from the observations of G8.68-0.37 in Pillai et al. 2007). This is lower than typical abundances found in young massive star formation regions but increasing the abundance to several $10^{-8}$ does not affect the general results of the paper.

Models were constructed over a range of values for the five parameters (see Table 3). Radiative transfer modeling was then used to generate synthetic data cubes for the $\mathrm{NH}_{3}(1,1)$, $(4,4)$, and $(5,5)$ emission. These were then convolved with twodimensional Gaussian profiles at a spatial scale corresponding to the resolution (synthesized beam) of the Longmore et al. (2007) observations. The synthetic spectra at each transition and position were fit to the observed spectra at three positions and reduced- $\chi^{2}$ values were returned for the goodness-of-fit. The first position was located at the peak of the submillimeter continuum emission $\left(\alpha_{J 2000}=18: 06: 23.47, \delta_{J 2000}=-21: 37: 7.6\right)$, then in radial steps of $\sim 10^{\prime \prime}$ (the synthesized beam size) at $\alpha_{J 2000}=18: 06: 23.83, \delta_{J 2000}=-21: 37: 15.6$ and $\alpha_{J 2000}=$ 18:06:24.33, $\delta_{J 2000}=-21: 37: 21.6$. While the $\mathrm{NH}_{3}$-integrated intensity emission shows a similar extent to the single-dish continuum emission, the $\mathrm{NH}_{3}$ morphology is extended along the

\footnotetext{
7 See Keto (1990) for a description of the MOLLIE code, Keto et al. (2004) for a description of the line fitting and Carolan et al. (2009) and Keto \& Zhang (2010) for recent examples of work using the code.
} 

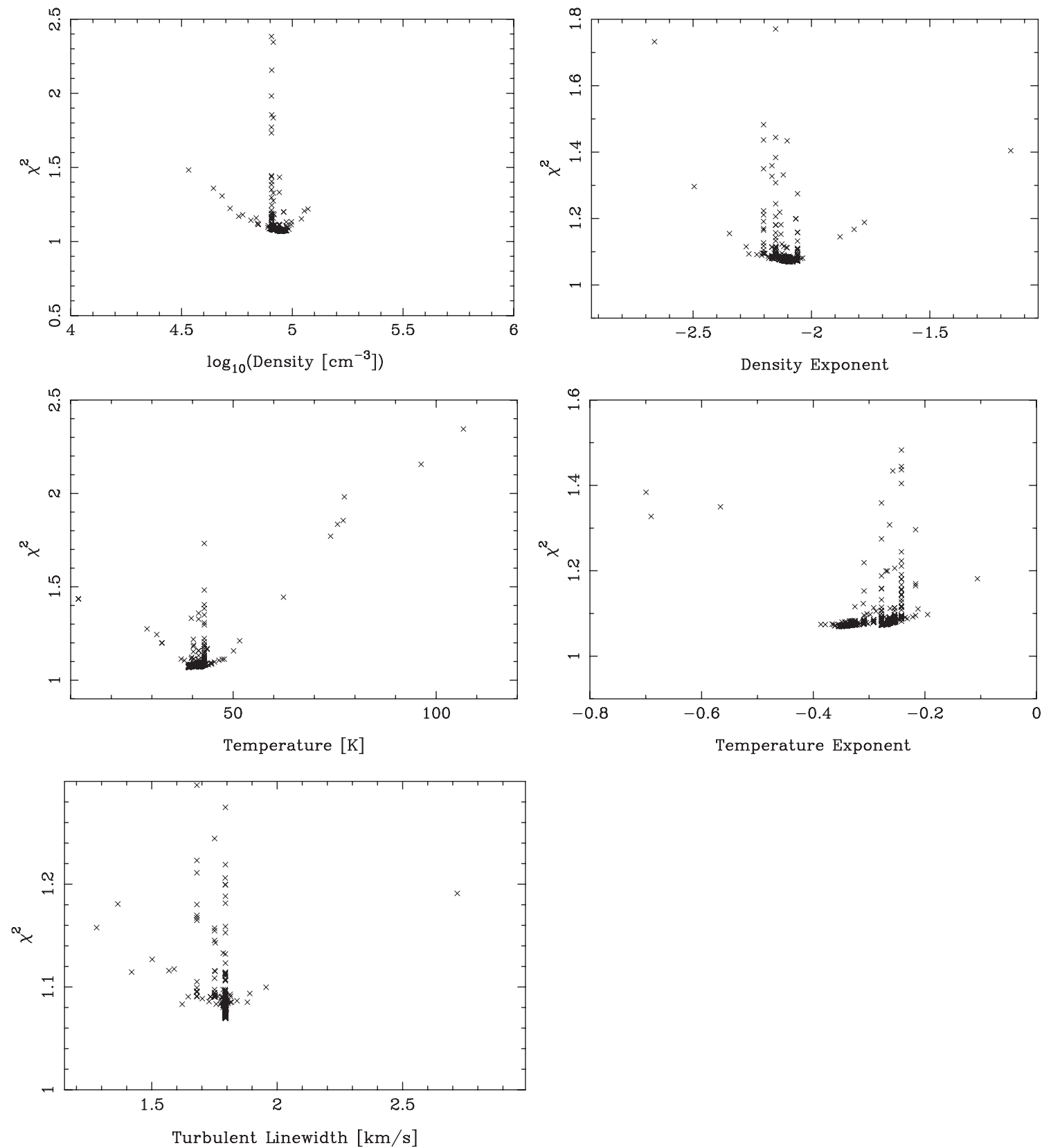

Figure 7. Reduced- $\chi^{2}$ values from the simulated annealing process to find the best-fit model to the Longmore et al. (2007) data (see Section 4.3.2). Each of the crosses shows the physical parameters (density, density exponent, temperature, temperature exponent, and turbulent linewidth) from one of the 10,000 models, and the resulting reduced $-\chi^{2}$ value for that particular model.

outflow axis. As $\mathrm{NH}_{3}$ is known to be affected by outflow interactions (Zhang et al. 2007b), it is possible that the $\mathrm{NH}_{3}$ emission may be affected along this axis. Therefore, the radial direction was chosen to be that perpendicular to the molecular outflow (see Section 4.2 and Figures 2 and 5) to minimize any potential contamination.

Simulated annealing with 10,000 models was used to search through the five-dimensional parameter space to minimize $\chi^{2}$ and find the best-fit model. Figure 7 shows the reduced- $\chi^{2}$ values for the range of parameter space covered by one such run through the simulated annealing process. This method is inherently robust against becoming trapped in local, rather than global minima in parameter space. However, to determine the robustness of the best-fit model we ran the fitting 20 times with widely separated initial start values and increments. The results are similar to those in Figure 7 . Table 3 lists the range of parameter space covered in the fitting process and the resulting best-fit values. Figure 8 shows the Longmore et al. (2007) $\mathrm{NH}_{3}(1,1),(4,4)$, and $(5,5)$ spectra from each of the three positions overlaid with the synthetic spectra at the same position from the best-fit model.

\subsection{3. $\mathrm{CH}_{3} \mathrm{CN}$ modeling}

Emission from the $\mathrm{CH}_{3} \mathrm{CN} J=12-11$ transition ( $\sim 220.7 \mathrm{GHz})$ is detected in the $K=0-7$ components $^{8}$ in the $230 \mathrm{GHz}$ compact-configuration observations. The emission is unresolved in the $3^{\prime \prime} \times 2^{\prime \prime}$ beam and at the peak of the millimeter continuum emission, encompassing the location of MM1 to MM3. Physical parameters of the gas at this spatial scale 8 Upper energy levels for $K=0-7$ components are $69,76,97,133,183,246$,
325 , and $417 \mathrm{~K}$, respectively. 

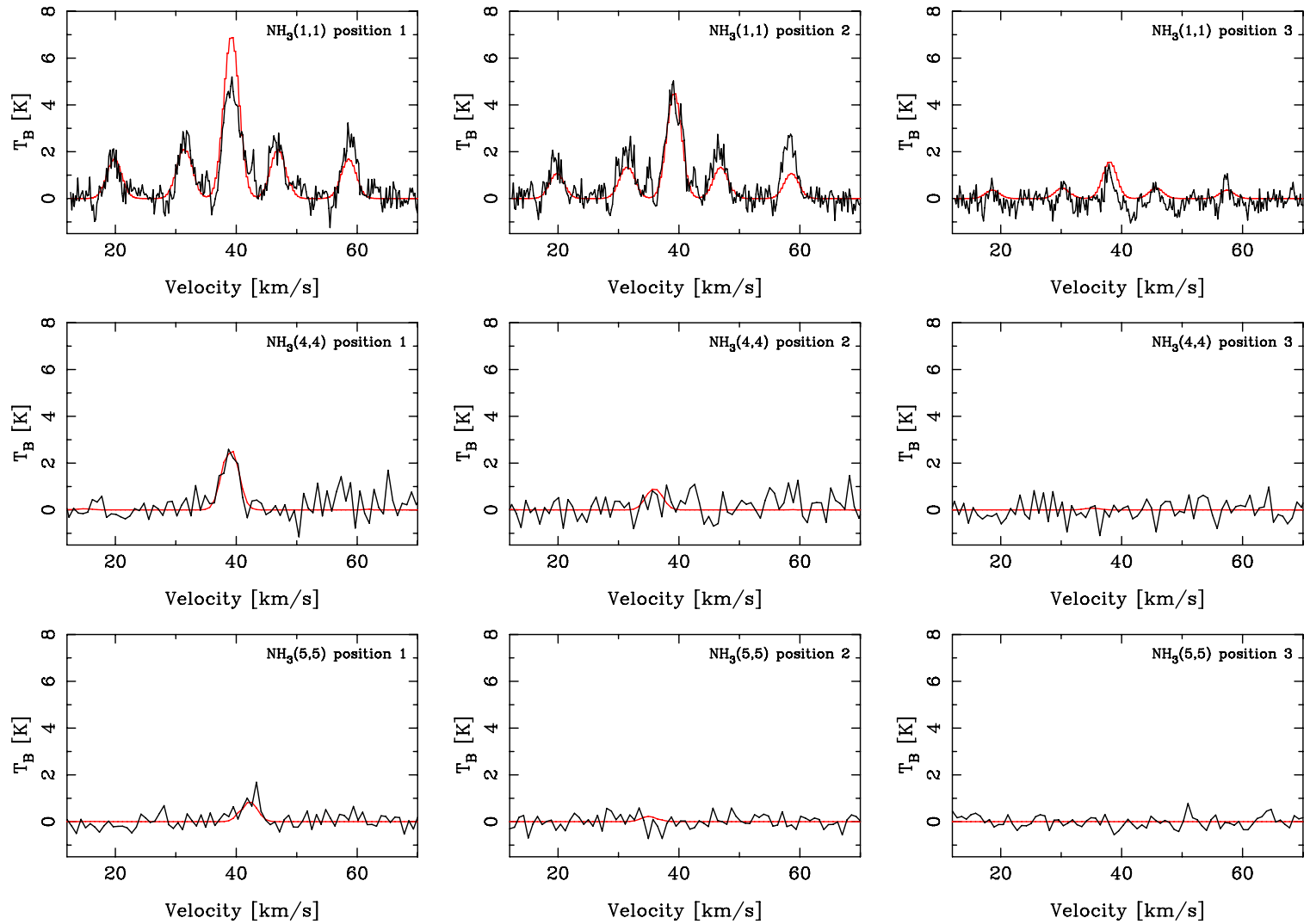

Figure 8. Results of the $\mathrm{NH}_{3}$ radiative transfer modeling outlined in Section 4.3.2. Longmore et al. (2007) spectra are shown in black, overlaid with the best-fit model spectra in red. Each column shows spectra taken from a single position on the sky. The top, middle, and bottom rows are the $\mathrm{NH}_{3}(1,1),(4,4)$, and $(5,5)$ transitions, respectively. The positions were selected to be spaced by roughly one synthesized beam in the direction perpendicular to the molecular outflow (see Sections 4.2 and 4.3.2) to minimize potential contamination from gas affected by the outflow.

(A color version of this figure is available in the online journal.)

Table 3

$\mathrm{NH}_{3}$ Radiative Transfer Modeling Parameters

\begin{tabular}{lrrr}
\hline \hline \multirow{2}{*}{ Parameter } & \multicolumn{2}{c}{ Search range } & Best-fit \\
\cline { 2 - 3 } & Min & Max & Value \\
\hline $\log \left(\rho_{1 / 2}\left(\mathrm{~cm}^{-3}\right)\right)$ & 3.0 & 8.0 & 4.98 \\
$\kappa_{\rho}$ & 0.5 & 4.0 & 2.08 \\
$T_{1 / 2}(\mathrm{~K})$ & 10.0 & 400.0 & 41.5 \\
$\kappa_{T}$ & 0.1 & 2.5 & 0.35 \\
$\Delta V_{\mathrm{NT}}\left(\mathrm{km} \mathrm{s}^{-1}\right)$ & 0.5 & 3.0 & 1.79 \\
\hline
\end{tabular}

Notes. A spherically symmetric model of radius $0.58 \mathrm{pc}$ was chosen based on the extent/morphology of the $\mathrm{NH}_{3}(1,1)$ emission. The temperature, $T_{K}$, and density, $\rho$, were parameterized with radius, $r$, as power laws: $\rho(r)=\rho_{1 / 2} r^{-\kappa_{\rho}}$ and $T_{K}(r)=T_{1 / 2} r^{-\kappa_{T}}$, where $\rho_{1 / 2}$ and $T_{1 / 2}$ are the density and temperature at the half-radius point, $0.29 \mathrm{pc}$. An additional constant velocity component, $\Delta V_{\mathrm{NT}}$, was included to model non-thermal support (e.g., from turbulence), resulting in a five-parameter model for the cloud. The second and third columns show the range of parameter space the models searched through. The final column gives the best-fit model to the L07A data after $\chi^{2}$ minimization of 10,000 models using simulated annealing to search through the five-dimensional parameter space. Full details of the modeling are given in Section 4.3.2.

were estimated by a $\chi^{2}$ minimization fitting of the spectrum, solving for the optical depth assuming LTE conditions. With a collisional rate of $10^{-8} \mathrm{~cm}^{3} \mathrm{~s}^{-1}$ (Green 1986) and Einstein $A$ coefficient of $10^{-4} \mathrm{~s}^{-1}$, the transition has a nominal critical density of $10^{4} \mathrm{~cm}^{-3}$ so the excitation should be collisionally dominated in densities typical of massive star-forming cores. The observed spectrum and resultant fit are shown in Figure 9.
While the single-component model clearly fits the data well, the increasing linewidth and slight excess of emission in the high$K$ versus low- $K$ components means that the emission probably has more than one component, and the gas potentially has a temperature and density gradient. However, the signal-to-noise ratio of the higher $K$-components is not sufficient to constrain more detailed modeling. The best-fit temperature including all $K$-components is $200 \mathrm{~K}$ which was robust to the $10 \%$ level under variation of the initial fitting estimates and number of free parameters. Fitting only the $K=0-4$ components reduces the temperature to $100 \mathrm{~K}$ but gives a much poorer result for the higher $K$ components. The best-fit $\mathrm{CH}_{3} \mathrm{CN}$ column density is $10^{16} \mathrm{~cm}^{-2}$. Given typical abundances (Wilner et al. 1994; Zhang et al. 1998, 2007a; Hatchell et al. 1998; Chen et al. 2006; Leurini et al. 2007), the total $\mathrm{H}_{2}$ column density at this size scale is at least $10^{24} \mathrm{~cm}^{-2}$ and possibly significantly larger. For the above models, the filling factor implies a size of 0.8 for the $\mathrm{CH}_{3} \mathrm{CN}$ emission region.

\subsubsection{Model Consistency}

We now compare the results of the modeling outlined in Sections 4.3.1, 4.3.2, and 4.3.3 to check for consistency. At larger spatial scales, the results of the continuum and $\mathrm{NH}_{3}$ modeling agree well, suggesting that the simple spherical model with power-law temperature and density gradient is a good approximation at this spatial scale. However, the fit to the SMA continuum data in Figure 6 shows the assumption of spherical symmetry breaks down at angular scales below a few arcseconds-corresponding to the size at which the extended 


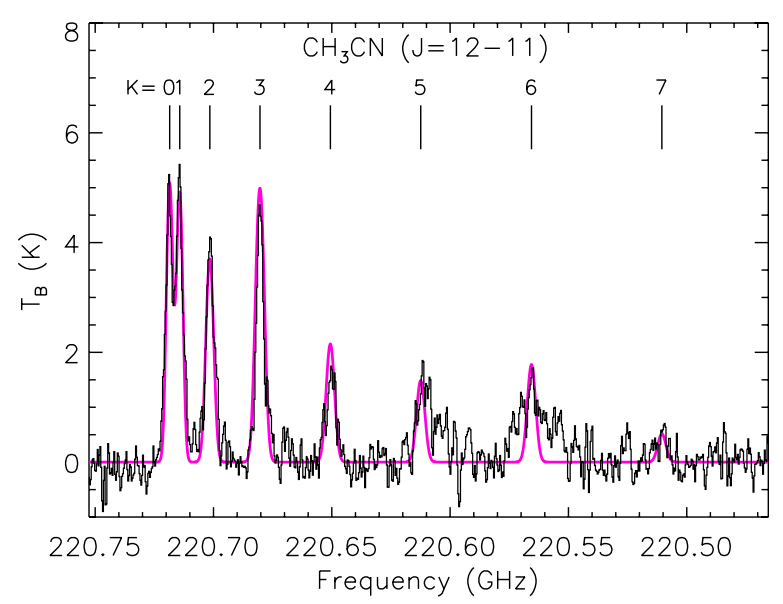

Figure 9. $\mathrm{CH}_{3} \mathrm{CN}(J=12-11)$ spectrum from the $230 \mathrm{GHz}$, compactconfiguration SMA data at the position of peak continuum emission (black) overlaid with the single-component LTE model fit as outlined in Section 4.3.3. The $K$-components are labeled above the spectrum.

(A color version of this figure is available in the online journal.)

configuration resolves the emission into the three cores, MM1 to MM3. In the subsequent discussion section, we refer to this radius of $\sim 0.05 \mathrm{pc}$ as the "minimum reliable radius" for the power-law density and temperature approximation.

Given the poorer resolution $\left(\geqslant 8^{\prime \prime}\right)$, the observed $\mathrm{NH}_{3}$ emission will be dominated by gas at much larger spatial scales. However, extrapolating the temperature and density gradient derived from the $\mathrm{NH}_{3}$ data down to $0^{\prime \prime} .8$ (the best-fit size of the $\mathrm{CH}_{3} \mathrm{CN}$ emission) predicts a temperature of $100 \mathrm{~K}$ and density of $2 \times 10^{7} \mathrm{~cm}^{-3}$, which matches well with properties derived from the $\mathrm{CH}_{3} \mathrm{CN}$ data when only fitting the lower $K$-components. The excess emission and linewidth in the higher $\mathrm{CH}_{3} \mathrm{CN} \mathrm{K}$-components point to the density and temperature gradient continuing at even smaller spatial scales. In summary, modeling of the three different data sets produces a coherent picture of the density and temperature profile of G8.68-0.37.

\section{MASSES OF MM1 TO MM3}

We calculate the mass of MM1 to MM3 using the temperatures derived from the $\mathrm{NH}_{3}$ and $\mathrm{CH}_{3} \mathrm{CN}$ data $-\sim 100 \mathrm{~K}$ at the radius encompassing all three components and likely increasing to $\sim 200 \mathrm{~K}$ at smaller radii. Without independent temperature measurements of each component, the plausible mass range of MM1 to MM3, assuming standard dust properties and following Kauffmann et al. (2008), is then 7-14 $M_{\odot}, 5-9 M_{\odot}$, and 2-5 $M_{\odot}$, respectively. This is a small fraction of the total mass of $1500 M_{\odot}$ derived from the single-dish observations (Hill et al. 2010).

\section{DISCUSSION}

We now return to the original focus of the paper to investigate the case for thermal feedback affecting fragmentation in this young massive protocluster.

We consider a simplistic scenario where, at $t=t_{0}$, the region initially had conditions similar to those of IRDCs: $T_{0} \sim 10-20 \mathrm{~K}$ and $n_{0} \sim 10^{4} \mathrm{~cm}^{-3}$. A first generation of stars formed from this gas and began to heat up the surrounding environment. Some time later, at $t=t_{\text {frag }}$, the more massive cores we observe with the SMA formed from thermal fragmentation of the gas. The gas temperature and density at $t_{\text {frag }}$ were $T_{\text {frag }}$ and $n_{\text {frag }}$. Assuming purely thermal fragmentation, this gas temperature and density set the mass $\left(M_{\text {frag }}\right)$ and separation $\left(\lambda_{\text {frag }}\right)$ of the resulting fragments. Some further time later, at $t=t_{\text {now }}$, we observed the region and have determined the present-day temperature $\left[T_{\text {now }}(\mathrm{r})\right]$ and density $\left[\rho_{\text {now }}(\mathrm{r})\right]$ as a function of radius from $r=0.05$ to $0.58 \mathrm{pc}$ and the current mass $\left(M_{\text {now }}\right)$ and separation $\left(\lambda_{\text {now }}\right)$ of cores MM1 to MM3 resolved on smaller scales.

In the following sections, we use these observational results to infer the possible conditions at $t_{\text {frag }}$ (i.e., $T_{\text {frag }}, \rho_{\text {frag }}, \lambda_{\text {frag }}$, and $M_{\text {frag }}{ }^{9}$ ). Given these inferred conditions, we then investigate the plausibility of different sources of feedback raising the gas temperature to the required $T_{\text {frag. }}$. In this way, we aim to assess whether thermal feedback may have affected fragmentation.

In Section 6.1, we start with the assumption that the present-day density profile and core mass/separations are similar to those at the time of fragmentation-so $\rho_{\text {now }}=\rho_{\text {frag }}$, $\lambda_{\text {now }}=\lambda_{\text {frag }}$, and $M_{\text {now }}=M_{\text {frag }}$. In Section 6.2, we relax these assumptions.

$$
\begin{aligned}
& \text { 6.1. Thermal Fragmentation Assuming } \rho_{\text {now }}=\rho_{\mathrm{frag}} \text {, } \\
& \qquad \begin{array}{c}
\lambda_{\text {now }}=\lambda_{\text {frag }} \text {, and } M_{\text {now }}=M_{\text {frag }} \\
\text { 6.1.1. Inferring } T_{\text {frag }}
\end{array}
\end{aligned}
$$

Assuming that MM1 to MM3 have not moved since fragmentation, their relative positions in Figure 2 then correspond to their fragmentation separations, $\lambda_{\text {frag }}$, or Jeans length, projected on to a two-dimensional plane. The measurement uncertainty in the projected separations is small-of order a few percent for the significance of the MM1 to MM3 detections (see, e.g., Fomalont 1999, Equation (14-5)), or calculated from the rms phase noise, $\Delta \phi$, and synthesized beam, $\theta_{B}$ (positional uncertainty $\left.\sim \Delta \phi \theta_{B} / 2 \pi\right)$. The major systematic uncertainty is the distance to the region, which we estimate to be $\sim 20 \%$. As the measured separations are in projection, they underestimate the real separations. We try to quantify this underestimation through geometrical arguments. Making the reasonable assumption that MM1 to MM3 lie within the volume encompassed by $r<0.05 \mathrm{pc}$ (as opposed to lying at much larger radii and only seen in projection toward the center), the largest underestimate will occur where a given pair lie at opposite sides (i.e., front versus back) of the $r<0.05$ pc volume. Assuming MM1 lies near the center, the maximum separation is then $0.05 \mathrm{pc}$. The projected separations then underestimate the real separations by $\leqslant 40 \%$. Any underestimate in separation will be reflected in an underestimate of $T_{\text {frag }}$.

Completeness is another potential observational bias in determining $\lambda_{\text {frag }}$. If we are missing lower-mass fragments which fall below the detection limit, for example, the distance from MM1 to MM3 to the nearest fragment may be lower than the measured projected separations. However, from the sensitivity of the SMA observations ( $\sigma_{\mathrm{rms}} \sim 1-2 \mathrm{mJy}$ ), any isolated fragments above $\sim 1 M_{\odot}$ should be detected by their dust continuum emission. This $1 M_{\odot}$ limit is equal to the global Jean's mass for the physical conditions typical of cold IRDCs prior to the formation of any stars. A more in-depth discussion of the potential existence of a population of pre-existing but undetected lower-mass stars is given in Section 6.1.2.

A final concern in determining $\lambda_{\text {frag }}$ might be that MM1 to MM3 are comprised of multiple, unresolved dust continuum peaks. For example, $\sim 0^{\prime} .2$ angular resolution observations toward similar regions at a similar distance and evolutionary

\footnotetext{
$9 \lambda_{\text {frag }}$ and $M_{\text {frag }}$ are equivalent to the Jeans length and Jeans mass, respectively.
} 
stage resolve multiple (proto)stars with linear separations down to $1700 \mathrm{AU}$ (Longmore et al. 2006). However, such systems would correspond to multiple stars forming within a single fragment, rather than reflecting the spatial distribution of fragments at $t_{\text {frag. }}$.

The uncertainties in the mass of MM1 to MM3, and hence $M_{\text {frag }}$, are far larger than those of $\lambda_{\text {frag }}$. In addition to the large uncertainty introduced by the lack of independent temperature measurements for MM1 to MM3 (see Section 5), there are major systematic uncertainties in deriving gas masses from thermal dust emission-e.g., dust properties, distance, ${ }^{10}$ gas-to-dust ratio, etc.

The density distribution is constrained at the larger spatial scales by the $\mathrm{NH}_{3}$ modeling (Section 4.3.2) and at arcsecond scales by the $\mathrm{CH}_{3} \mathrm{CN}$ modeling (Section 4.3.3). As discussed in Section 4.3.4, these independently predict a density of $\sim 2 \times 10^{7} \mathrm{~cm}^{-3}$ at the size scales of MM1 to MM3. This provides an upper limit to $\rho_{\text {frag }}$ as the rest of the volume containing MM1 to MM3 is likely to be at a lower density. As a more realistic estimate of $\rho_{\text {frag }}$, the density profile derived in Section 4.3 predicts an average density of $8 \times 10^{6} \mathrm{~cm}^{-3}$ within a volume of $r<0.05$ pc (the radius encompassing MM1 to MM3).

Figure 10 encapsulates the above uncertainties and illustrates how these affect the inferred fragmentation temperature. The solid lines show the expected $T_{\text {frag }}$ as a function of density for Jeans lengths of 0.05 and $0.03 \mathrm{pc}$, corresponding to upper and lower limit estimates of the MM1 to MM3 separations. The dotted lines show the expected $T_{\text {frag }}$ as a function of density for Jeans masses of 3 and $10 M_{\odot}$, corresponding to reasonable estimates of the MM1 to MM3 masses. This shows that for the predicted densities, the fragmentation temperature must be at least $100 \mathrm{~K}$.

We now consider the plausibility of different sources of feedback raising the gas temperature to this required $T_{\text {frag }}$.

\subsubsection{The Case for Heating by Pre-existing, Unobserved, Embedded Lower-mass Stars}

From the sensitivity of the SMA observations $\left(\sigma_{\mathrm{rms}} \sim\right.$ 1-2 mJy), any isolated dust continuum fragments above $\sim 1 M_{\odot}$ should be detected, but the SMA would not detect unembedded stars. The Spitzer observations (e.g., Figure 2) would detect stars, but not if they were very deeply embedded. Due to spatial filtering, interferometers are only sensitive to density contrasts, so it is possible that the observations are missing a population of uniformly distributed, embedded lower-mass stars.

To assess the feasibility that pre-existing and unobserved stars are responsible for heating G8.68-0.37, we investigate what stellar population would be required to generate the required $100 \mathrm{~K}$ thermal fragmentation temperature. We take the simple approach of calculating the effect of heating by individual stars of different mass and luminosity to find the radius out to which they will heat the gas to $100 \mathrm{~K}$. Given this radius, we then determine the minimum number of these stars uniformly spaced within the total spherical volume of radius $0.05 \mathrm{pc}$ necessary to raise the temperature to $100 \mathrm{~K}$.

Assuming that the stars and surrounding dust are in radiative equilibrium ${ }^{11}$ and the opacity can be approximated by a power law of wavelength proportional to $\lambda^{-p}$, a star with temperature

\footnotetext{
10 The uncertainty in mass is proportional the uncertainty in distance, $\Delta D$, squared, rather than linearly proportional to $\Delta D$ as is the case for the uncertainty in the measured separations.

11 For example, each radius is considered as a geometrically thin and optically thick shell with no opacity between it and the star.
}

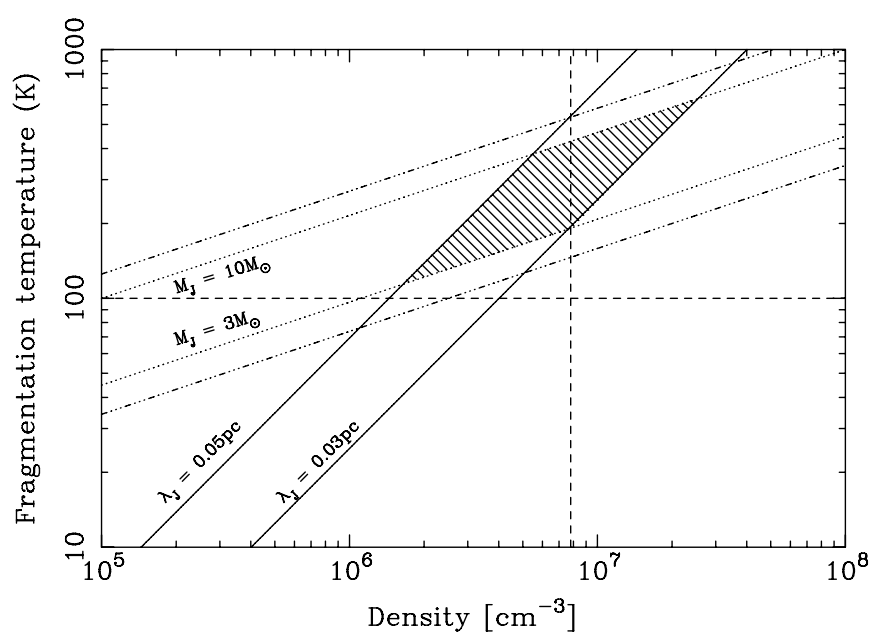

Figure 10. Encapsulating the observational uncertainties in the measured separations and mass of MM1 to MM3 to illustrate how these affect the inferred fragmentation temperature. The solid lines show the expected $T_{\text {frag }}$ as a function of density for Jeans lengths of 0.05 and $0.03 \mathrm{pc}$, corresponding to upper and lower limit estimates of the MM1 to MM3 separations. The dotted lines show the expected $T_{\text {frag }}$ as a function of density for Jeans masses of 3 and $10 M_{\odot}$, corresponding to reasonable estimates of the MM1 to MM3 masses. The dot-dashed lines illustrate the most extreme mass range given the observational uncertainties. The vertical dashed line shows the average density within a volume of $r<0.05 \mathrm{pc}$ (the radius encompassing MM1 to MM3) of $7.8 \times 10^{6} \mathrm{~cm}^{-3}$ as a reasonable estimate for the fragmentation density. The shaded region illustrating the observational limits shows that the fragmentation temperature is at least $100 \mathrm{~K}$ (the horizontal dashed line). If the density were lower in the past (for example, if the region were undergoing global infall), the fragmentation temperature would also be lower.

$T_{\star}$ and radius $R_{\star}$ will raise the dust to temperature $T_{d}$ at a radius $r_{d}\left(r_{d} \gg R_{\star}\right)$, given by $r_{d} \simeq\left(R_{\star} / 2\right)\left(T_{d} / T_{\star}\right)^{-(4+p) / 2}$ (Lamers \& Cassinelli 1999, Equation (7.37)). We adopt $p=1.5$ based on the opacities determined from the MRN (Mathis et al. 1977) grain-size distribution between wavelengths of $0.1 \mu \mathrm{m}$ and 1 mm (Kruegel \& Siebenmorgen 1994). To investigate the case of maximal heating, the central stars are assumed to have reached the main sequence. This provides an upper limit to the stellar effective temperature because protostars on their Hayashi tracks are generally cooler (Cox 2000, Table 15.14). Figure 11 shows the resulting dust temperature as a function of radius from stars of mass $=0.8,1.2,1.8,2.5,5$, and $10 M_{\odot}$, overlaid with the best-fit model profile determined in Section 4.3. Main-sequence stars of $0.8,1.2$, and $1.8 M_{\odot}$ will heat dust to $\geqslant 100 \mathrm{~K}$ at radii $\leqslant 3.5 \times 10^{-4}, 1.0 \times 10^{-3}$, and $3.5 \times 10^{-3} \mathrm{pc}$, respectively. To heat the volume of radius $0.05 \mathrm{pc}$ to $100 \mathrm{~K}$ in this way therefore requires uniformly distributing these stars by the above separations throughout the volume. The minimum number of stars this implies $\left(2.9 \times 10^{6}, 1.25 \times 10^{5}\right.$, and $\left.2.9 \times 10^{3}\right)$ within a radius of $0.05 \mathrm{pc}$ leads to total stellar masses far in excess of the total mass of gas available. We therefore conclude the number of stars required to heat the gas is unfeasibly large.

At the earliest evolutionary stages, the luminosity from lowmass stars can be dominated by accretion rather than nuclear burning. We now consider the effect of heating due to accretion luminosity. As most of the infalling particles' potential energy will be released at radii close to the protostar, the luminosity can be approximated as arising from a point source. Following similar assumptions about the dust properties, etc., as before, the dust temperature as a function of radius can be solved analytically (e.g., Scoville \& Kwan 1976; Garay \& Lizano 1999, Equation (11)). The left-hand panel of Figure 12 shows the dust temperature as a function of radius for central heating 


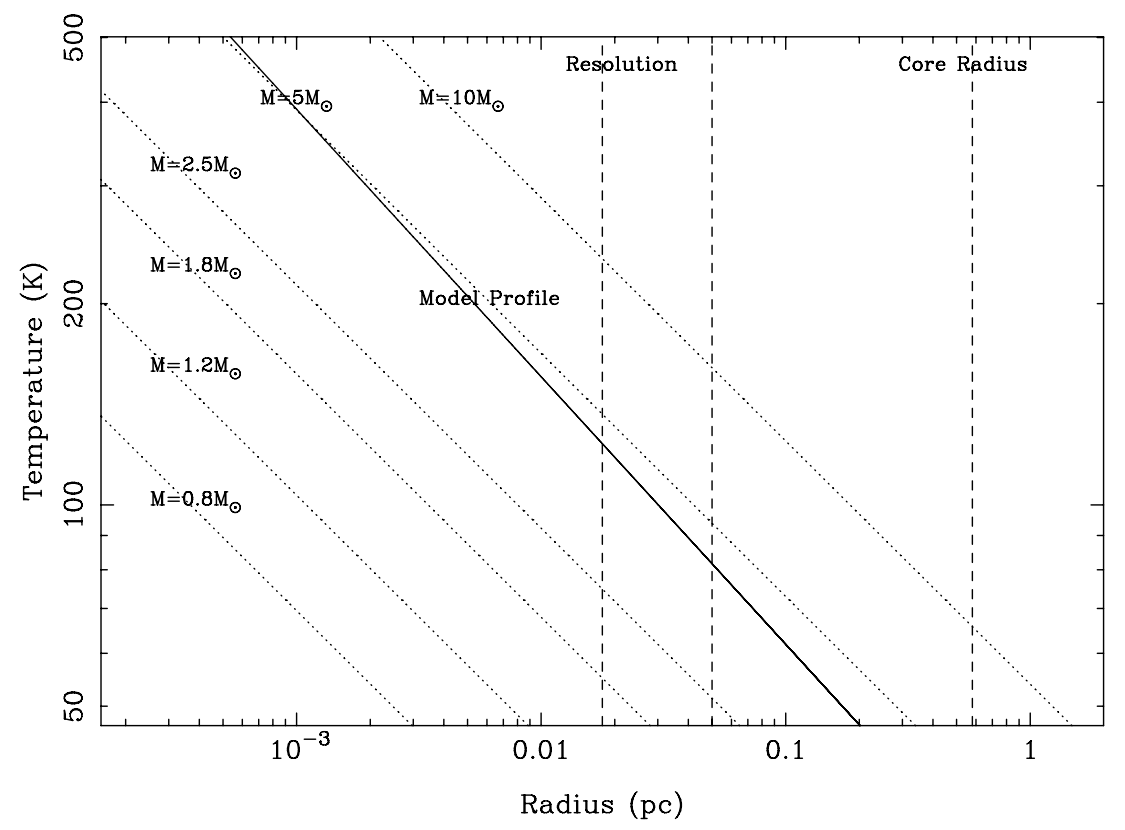

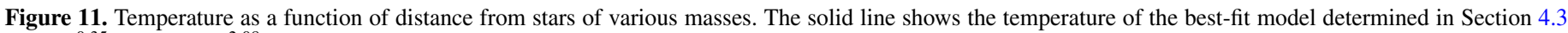

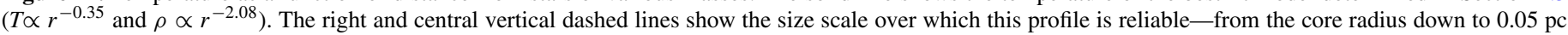

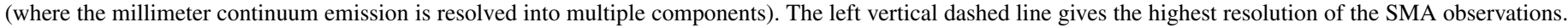

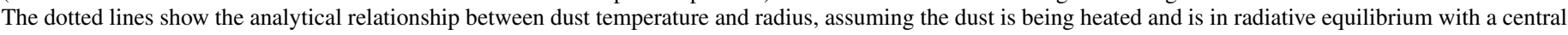

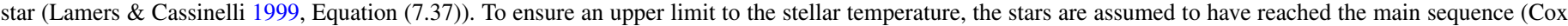
2000, Table 15.14). Temperature profiles are shown for stars of mass $=0.8,1.2,1.8,2.5,5$, and $10 M_{\odot}$.

sources with luminosities from 1 to $10^{5} L_{\odot}$. This range was chosen to include the expected luminosity from accreting lowmass stars (which are dominated by accretion luminosity) to $\mathrm{O}$ stars dominated by stellar luminosity. The right-hand panel of Figure 12 shows the radius at which central heating sources of a given luminosity will heat the surrounding gas up to $\geqslant 100 \mathrm{~K}$. Following the same line of argument as before, we can investigate how many sources are required to heat the $100 M_{\odot}$ volume of radius $0.05 \mathrm{pc}$ (the mass determined from the modeling that lies within the radius encompassing MM1 to MM3) to $100 \mathrm{~K}$ as observed in G8.68-0.37. Froebrich (2005) shows that isolated Class 0 cores (which as the youngest protostars should have the highest accretion rates) typically have luminosities $\leqslant 10 L_{\odot}$. Again, an unfeasible number of low-mass stars are needed to heat the gas to the required temperature, even allowing for the additional heating from accretion luminosity. Assuming the higher infall rates in a massive protocluster environment give rise to larger accretion luminosities does not solve the problem either. The total luminosity required would be larger than the present-day measured bolometric luminosity of $1.9 \times 10^{4} L_{\odot}$.

Comparing the Lamers \& Cassinelli (1999) temperature profiles to those of Garay \& Lizano (1999), ${ }^{12}$ we note that the former are higher by factors of a few. The Garay \& Lizano (1999) formulation appears closer to observed profiles (e.g., Keto \& Zhang 2010, and the gas temperatures derived from our own observations; Section 4.3) so we consider the Lamers \& Cassinelli (1999) formulation (e.g., Figure 11) as an upper limit to the temperature.

The discrepancy between these two methods does not affect the conclusion from the above analysis. A large population of low-mass stars would not be able to heat the gas to the $100 \mathrm{~K}$

12 We compared the two analytic solutions by taking the known radii and effective temperatures of ZAMS stars of a given luminosity. thermal fragmentation temperature required to reproduce the observed separations and masses of cores MM1 to MM3. This is consistent with the models of Offner et al. (2009) which show that low-mass stars have a negligible effect on the overall cloud heating. Repeating the above calculations but imposing more realistic limits - that the total stellar mass is not larger than the available gas mass and the total luminosity is not larger than the measured bolometric luminosity-gives an upper limit to the temperature provided by low-mass stars of $\sim 40 \mathrm{~K}$.

\subsubsection{The Case for Heating by an Early-B Star}

The calculations in the previous section show that low-mass stars would not be able to heat the gas within a radius of $0.05 \mathrm{pc}$ to the temperature of $100 \mathrm{~K}$ required for the observed cores (MM1, MM2, and MM3) to have formed by thermal fragmentation. The solid line representing the best-fit model temperature profile in Figure 11 shows that this heating could be attributed to a single early B-type star rather than a larger number of low-mass stars. However, invoking an early B-type star as a source of thermal feedback to raise the gas temperature and suppress fragmentation (allowing cores as massive as MM1, MM2, and MM3 to form) is unsatisfactory as it invokes a circular argument-it does not solve the problem of what initially raised the temperature to suppress fragmentation into lowermass fragments which would have allowed the B star to form in the first place.

\subsection{Thermal Fragmentation Relaxing the Assumptions that $\rho_{\text {now }}=\rho_{\text {frag }}, \lambda_{\text {now }}=\lambda_{\text {frag }}$, and $M_{\text {now }}=M_{\text {frag }}$}

Having concluded that it is not possible for thermal feedback to have raised the gas temperature to $\geqslant 100 \mathrm{~K}$ if $\rho_{\text {now }}=\rho_{\text {frag }}$, $\lambda_{\text {now }}=\lambda_{\text {frag }}$, and $M_{\text {now }}=M_{\text {frag }}$, we consider how changes in the gas conditions from $t_{\text {frag }}$ to the present day could have affected the resulting fragments and look for observational predictions that these changes would imply. 

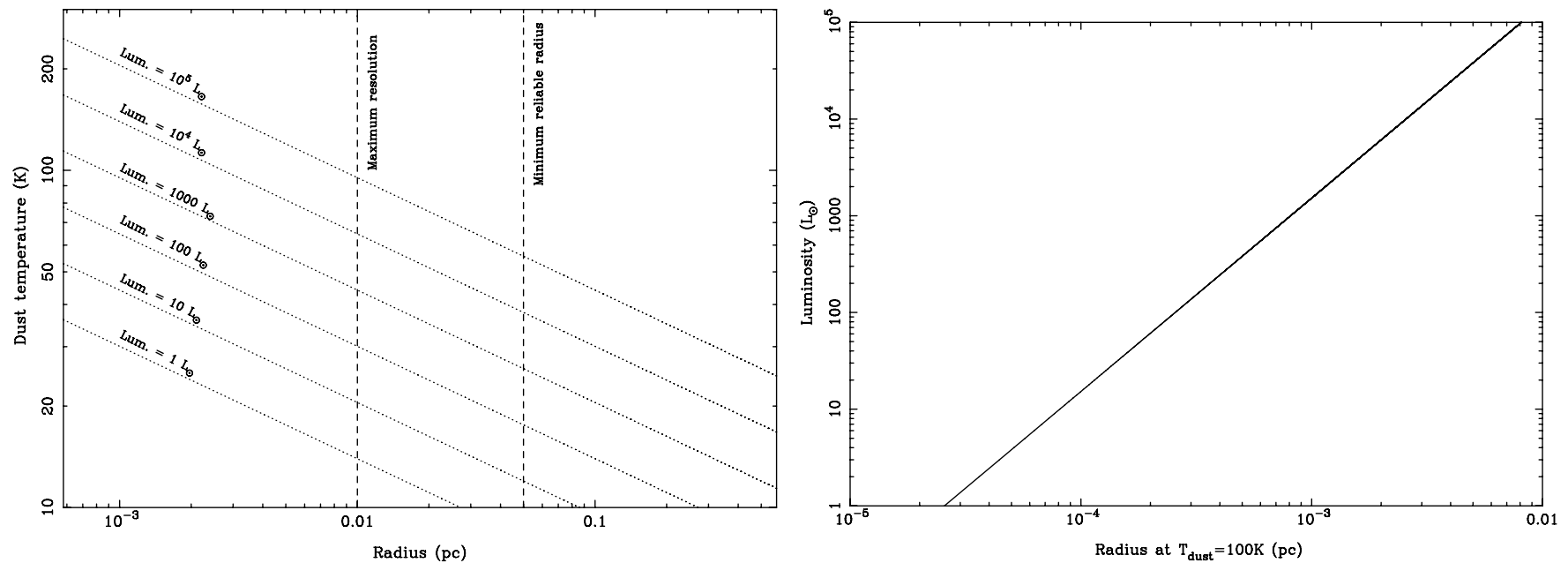

Figure 12. Left: dust temperature as a function of radius for central heating sources of different luminosity. The right and left vertical dashed lines show the radius where the millimeter continuum emission is resolved into multiple components and the highest resolution of the SMA observations, respectively. The dotted lines show the analytical relationship between dust temperature and radius, assuming the dust is being heated and is in radiative equilibrium with a central powering source of the listed luminosity (Scoville \& Kwan 1976, Equation (9)). Right: using the same analytical description in Scoville \& Kwan (1976), the solid line shows the source luminosity required to heat the gas to $\geqslant 100 \mathrm{~K}$ at the distance given on the $x$-axis.

As shown in Figure 10, the required fragmentation temperature would be lower than $100 \mathrm{~K}$ if the density at the time of fragmentation were lower than the present day-i.e., if $\rho_{\text {now }}>\rho_{\text {frag. }}$. In Section 6.2.1, we investigate the observational evidence for the large-scale infall of gas that this would imply. In Section 6.2.2, we consider how a lower density of gas at $t_{\text {frag }}$ would have affected the separations of MM1 to MM3 over time.

\subsubsection{The Case for Large-scale Infall}

As discussed in Section 1.1, the molecular line profiles in Purcell et al. (2009) provide observational evidence for largescale infall of gas in G8.68-0.37. Following the calculations in Walsh et al. (2006), the infall rate for G8.68-0.37 inferred from the $\mathrm{HCO}^{+}$and $\mathrm{HCN}$ spectra is $\sim 10^{-4} M_{\odot} \mathrm{yr}^{-1}$. This infall rate is significantly larger than typically measured toward lowermass star-forming cores but comparable to observations of other young high-mass star formation regions (Wu \& Evans 2003; Peretto et al. 2006, 2007; Walsh et al. 2006; Keto \& Klaassen 2008; de Wit et al. 2010).

Are these infall motions suggested by the molecular line observations consistent with the velocities expected in gravitational collapse? We estimate the dynamical state of the region by comparing the contribution from thermal and non-thermal support as a function of radius to the virial velocity, $V_{\text {virial }} \equiv\left(\frac{G M}{R}\right)^{1 / 2}$ (Stahler \& Palla 2005, Equation (3.20)) —a measure of how much kinetic energy is required to balance the gravitational potential. To convert from the measured one-dimensional velocity to the three-dimensional root mean square velocity, we use Rohlfs \& Wilson (2004), Equation (12.72). The virial velocity in Figure 13 is then calculated at each radius using the enclosed mass (derived from the model parameters in Section 4.3) inside that radius. The range of uncertainty in virial velocity (see, e.g., Elmegreen 1989) is illustrated by the hatched area between the dash-dot-dot lines. Figure 13 shows that (1) as expected the linewidth is dominated by the non-thermal contribution and (2) even the higher temperatures toward the center are insufficient to reach the required kinetic energy support for the cloud to be in equilibrium. Although undoubtedly oversimplifying the gas dynamical state (both support from magnetic fields and the surface terms in the virial equation are ignored, for example),

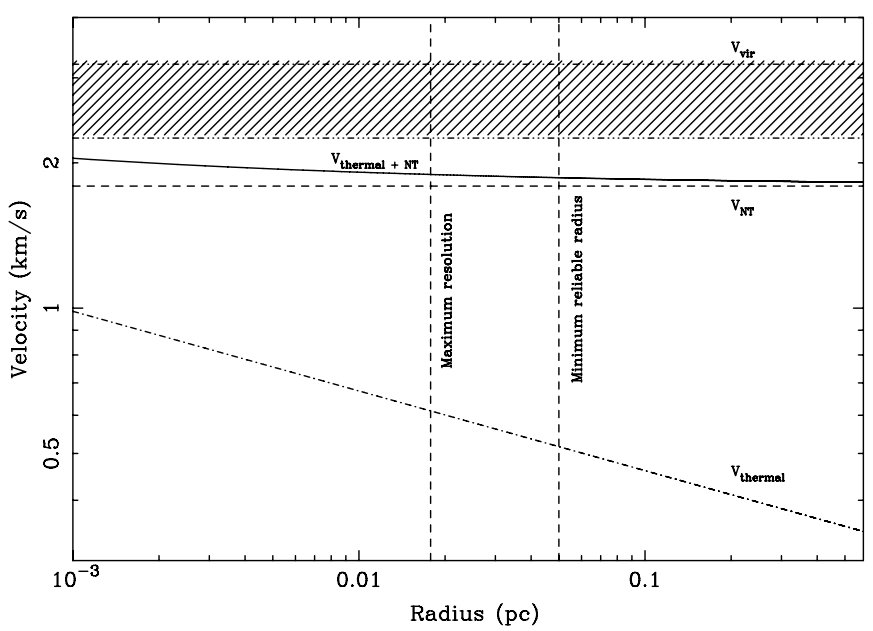

Figure 13. Comparison of non-thermal and thermal support in G8.68-0.37. The horizontal dashed line, indicated as $V_{\mathrm{NT}}$, shows the non-thermal contribution to the linewidth determined from the $\mathrm{NH}_{3}$ radiative transfer modeling in Section 4.3.2. The dot-dashed line, labeled $V_{\text {thermal }}$, shows the expected thermal contribution to the $\mathrm{NH}_{3}$ linewidth as a function of radius, determined from the temperature distribution of the best-fit model in Section 4.3. The solid line, $V_{\text {thermal+NT }}$, shows $V_{\mathrm{NT}}$ and $V_{\text {thermal }}$ added together in quadrature giving the expected total linewidth. Finally, the dash-dot-dot lines and enclosed hatched area give the expected range in virial velocity, $V_{\text {virial }} \equiv\left(\frac{G M}{R}\right)^{1 / 2}$ - a measure of how much kinetic energy is required to balance the gravitational potential of a cloud. The right and left vertical dashed lines show the minimum reliable radius for the assumed power-law density distribution and the maximum resolution of the SMA observations, respectively. This figure shows that (1) as expected the linewidth is dominated by the non-thermal contribution and (2) even the higher temperatures toward the center are insufficient to reach the required kinetic energy support for the cloud to be in equilibrium.

we find that the inward motions are generally consistent with gravitational contraction.

\subsubsection{Could the Cores Have Been More Widely Separated in the Past?}

If the entire star-forming cloud is globally contracting, then the density of the gas from which MM1 to MM3 fragmented could have been lower than measured today. The cores could initially have formed at larger separations and moved closer over time to reach their current locations. To test the feasibility of this 


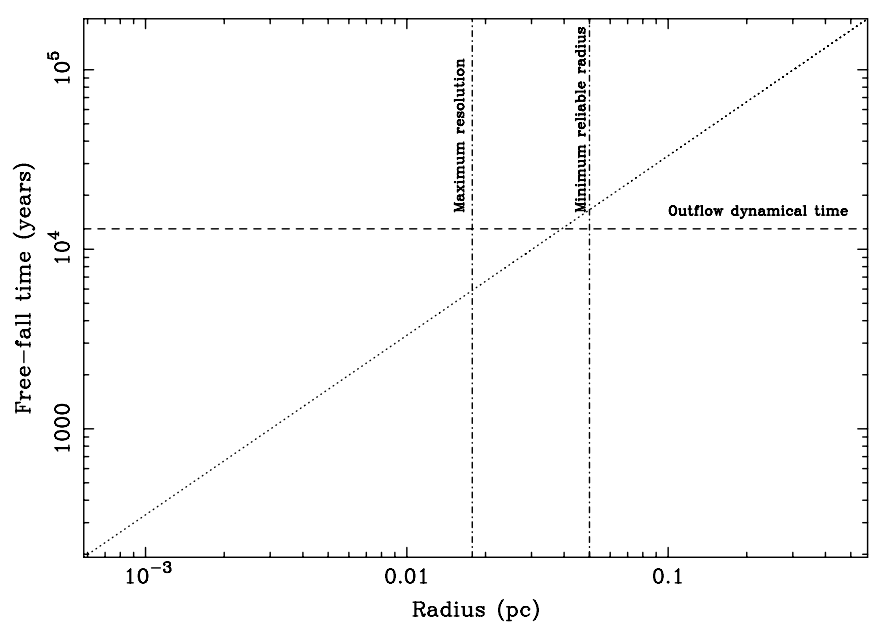

Figure 14. Dotted line shows the free-fall time, $t_{\mathrm{ff}} \equiv\left(\frac{3 \pi}{32 G \rho_{\mathrm{av}}}\right)^{1 / 2}$, as a function of radius, where $\rho_{\mathrm{av}}$ is the average density enclosed within a given radius determined from the enclosed mass within the radius on the horizontal axis. The right and left vertical dot-dashed line shows the minimum reliable radius for the assumed power-law density distribution and the maximum resolution of the SMA observations, respectively. The dynamical time of the outflow determined in Section 4.2 is shown as a dashed horizontal line. The free-fall time at the radius at which the core is observed to fragment is similar to the dynamical time of the outflow.

scenario, we can estimate how far these cores may have moved over a given time period and predict their resulting relative velocities. Assuming the fragmentation conditions were similar to those of IRDCs $\left(10^{5} \mathrm{~cm}^{-3}\right.$ and $\left.10-20 \mathrm{~K}\right)$, which are thought to be massive star formation regions at the earliest evolutionary stages, the Jeans length would have been $\sim 0.07 \mathrm{pc}$. To reach their current separations of $\sim 0.048 \mathrm{pc}$, the cores would have had to move $\sim 0.01 \mathrm{pc}$ toward each other.

We use the free-fall time to derive a lower limit on how long the cores may have been moving since they formed. Figure 14 shows the free-fall time, $t_{\mathrm{ff}} \equiv\left(\frac{3 \pi}{32 G \rho_{\mathrm{av}}}\right)^{1 / 2}$, of the gas in G8.68-0.37 as a function of radius, where $\rho_{\mathrm{av}}$ is the average density within each volume calculated from the enclosed mass within that radius. Taking $10^{4} \mathrm{yr}$ (the free-fall time at $0.05 \mathrm{pc}$ ) as a lower limit of the protocluster age and following Walsh et al. (2004), cores moving 0.01 pc would reach a velocity of $2.6 \mathrm{~km} \mathrm{~s}^{-1}$. Future molecular line observations with sufficient angular resolution to identify MM1 to MM3 should easily be able to discern such large relative velocities. The inferred proper motions of $\sim 60 \mu^{\prime \prime} \mathrm{yr}^{-1}$ are too small to detect.

\subsection{Considering Non-thermal Fragmentation}

In the preceding sections, we have used Jeans analysis to determine the mass and separation of core fragments, given the temperature and density of the surrounding environment. While the assumption of purely thermal support is undoubtedly an oversimplification - both turbulence and magnetic fields are also likely important as support mechanisms at the earliest stages of massive star formation (e.g., Zhang et al. 2009; Girart et al. 2009; Tang et al. 2009a, 2009b, 2010)—the relative importance of the different support mechanisms is not well understood at these spatial scales and for objects at such an early evolutionary stage. Without a direct measurement of the magnetic field toward G8.68-0.37, we make the assumption of equipartition between thermal, turbulent, and magneticfield support and assess how this affects the fragmentation temperature determined in Section 6.1.1. Returning to Figure 10, we used a lower limit fragmentation temperature of $100 \mathrm{~K}$ in the analysis of Section 6.1. If temperature, magnetic fields, and turbulence are in equipartition, the total energy is equivalent to a temperature of $300 \mathrm{~K}$. Figure 10 shows that $300 \mathrm{~K}$ is in fact a more realistic fragmentation temperature than the $100 \mathrm{~K}$ previously assumed. We therefore conclude that the results derived assuming purely thermal fragmentation are robust when including support from turbulence and magnetic fields.

Finally, we consider the assumption in the Jeans analysis that the gas is isothermal. Krumholz et al. (2007) find in their numerical models that at high column densities, where the gas becomes optically thick, the equation of state deviates from isothermal. The result is that fragmentation is suppressed at a lower temperature than would be expected from Jeans analysis. While this is difficult to test with observations of a single region, in future work we will look to compare the mass distribution of cores above and below the critical column density of $1 \mathrm{~g} \mathrm{~cm}^{-2}$, at which this effect is predicted to become important (Krumholz \& McKee 2008).

\subsection{Will the G8.68-0.37 Protocluster Form O Stars?}

In order to form an $\mathrm{O}$ star through direct collapse, a fragment would need to have a mass of at least the stellar mass times by the star formation efficiency-i.e., of order $100 M_{\odot}$ of gas, given typical star formation efficiencies (e.g., Lada \& Lada 2003). No matter what the fragmentation history of the region, none of the observed MM1 to MM3 cores has this much mass. Any feedback (if it exists/existed) was therefore not sufficient to create a fragment that can collapse to form an $\mathrm{O}$ star. In order to form an $\mathrm{O}$ star via accretion, these existing cores need to obtain additional mass from elsewhere. We now investigate whether G8.68-0.37 is likely to form $\mathrm{O}$ stars and, if so, how this might occur.

The total mass within $r<0.05$ pc (encompassing MM1 to MM3) predicted from the best-fit model density profile is $\sim 100 M_{\odot}$. MM1 to MM3 account for roughly a third of the mass, leaving a large reservoir of material inside this relatively small volume. If the measured infall rate (see Section 6.2.1) can be sustained for a few $10^{5} \mathrm{yr}$ and large-scale infall can feed mass to smaller spatial scales (as observed toward G20.08-0.14N by Galván-Madrid et al. 2009), it seems plausible that MM1 to MM3 may end up as $\mathrm{O}$ stars via continued accretion. However, without information of the gas velocities at $r<0.05 \mathrm{pc}$, it is not clear how MM1 to MM3 are coupled to the gas at this size scale and whether or not they will continue to gain mass. Several theoretical scenarios are postulated: continued accretion through a common rotating envelope or "disk" (e.g., Keto 2003, 2007; Krumholz et al. 2009); accretion from initially unbound cluster-scale gas (see, e.g., Smith et al. 2009); or fragmentationinduced starvation via gravitational instabilities in the accretion flow (Peters et al. 2010).

Considering the first scenario-that MM1 to MM3 may be fed by accretion through a common rotating envelope or disk-we do not detect molecular line emission in the highest angular resolution observations so cannot search for kinematic signatures of rotation at this spatial scale. However, it is interesting to note that MM1 to MM3 are oriented roughly in a plane perpendicular to the outflow. This is similar to the results of Fallscheer et al. (2009) toward 18223-3-another young high-mass protocluster, with similar global mass, infall, and outflow rates. They find that the millimeter continuum emission peaks are aligned perpendicular to the outflow and encompassed by a flattened, rotating entity of inward spiraling molecular 
gas. Although emission from a rotating envelope of 28,000 AU would have been resolved in the observations of G8.68-0.37, it is not seen. But such a large rotating envelope is not necessarily expected. A recent large survey to find high-mass accretion disk candidates (Beuther et al. 2009) found diameters of the rotating entities to be $\sim 10,000 \mathrm{AU}$, smaller than the unresolved bright molecular line emission in the SMA observations in this work.

The second scenario is similar to that described in Section 6.2.2 where the cores initially form with smaller masses and at larger radii. Over time they move closer under the influence of the global gravitational potential. In this picture, cores grow by accreting the diffuse surrounding unbound cluster-scale gas. With this in mind, it is interesting that the most massive observed millimeter continuum core, MM1, is found at the center of the gravitational potential (as predicted) where the infall would be greatest. However, other than searching for the relative velocity of the cores (see Section 6.2.2), it is difficult to test these predictions with observations of a single region. We are currently in the process of extending this analysis to larger samples of massive star formation regions (e.g., from the $\mathrm{NH}_{3}$ cores detected in the HOPS Galactic Plane survey Walsh et al. 2008).

In summary, we conclude that G8.68-0.37 may still proceed to form $\mathrm{O}$ stars, with large-scale infall and continued accretion at smaller scales feeding the observed millimeter cores. Observations to resolve the gas kinematics at $r<0.05 \mathrm{pc}$ are required to determine (1) how the millimeter cores and remaining gas are coupled at this size scale and (2) the mechanism through which accretion is taking place.

\section{CONCLUSIONS}

Combining multiple-configuration SMA continuum observations with modeling of the $\mathrm{NH}_{3}$ and $\mathrm{CH}_{3} \mathrm{CN}$ emission, we have determined the present-day (1) temperature/density as a function of radius and (2) mass/separation of cores, in the massive protocluster G8.68-0.37. We then used this to investigate whether feedback from low-mass stars can raise the protocluster gas temperature sufficiently to delay thermal fragmentation, allowing massive stars to form through direct collapse of highmass fragments.

From radii of $0.58 \mathrm{pc}\left(1.2 \times 10^{5} \mathrm{AU}\right)$ down to $0.05 \mathrm{pc}$ $\left(10^{4} \mathrm{AU}\right)$, we find that the data are well fit with the region having a power-law temperature and density of the form $T \propto r^{-0.35}$ and $\rho \propto r^{-2.08}$. At $r<0.05 \mathrm{pc}$, the assumption of spherical symmetry breaks down and the temperature/density range is calculated to be $100-200 \mathrm{~K}$ and $10^{6}-10^{7} \mathrm{~cm}^{-3}$, respectively. Within this radius of $0.05 \mathrm{pc}$, the SMA resolves the $1.2 \mathrm{~mm}$ continuum emission into three cores, MM1 to MM3, with separations of $6200 \mathrm{AU}$ and $9700 \mathrm{AU}$ and masses $10 \pm 3 M_{\odot}$, $7 \pm 2 M_{\odot}$, and $4 \pm 2 M_{\odot}$, respectively.

From the region's observed properties, we infer the conditions at the time the cores formed by fragmentation. Assuming the measured separations of MM1 to MM3 and the average density encompassing these cores $(r<0.05 \mathrm{pc})$ are representative of the physical conditions at fragmentation, this implies a thermal fragmentation temperature of at least $100 \mathrm{~K}$. We rule out a population of low-mass stars being able to provide this heating - an unfeasibly large number is required and the measured bolometric luminosity is orders of magnitude too small even when allowing for heating due to trapped accretion luminosity. The heating could instead be provided by the equivalent of a $5 M_{\odot}$ zero-age main sequence (ZAMS) star, but this is an unsatisfactory source of thermal feedback-it invokes a circular argument requiring the star to have raised the temperature to suppress fragmentation, before it formed. Alternatively, the required fragmentation temperature could be lower if the region were initially at a lower density, for example, if the cores formed farther apart or the region was undergoing global infall.

Whatever the fragmentation history, none of the observed cores has sufficient raw material to form an O star through direct collapse. Even if feedback may have suppressed fragmentation, it was not sufficient to halt it to this extent. If G8.68-0.37 is destined to form $\mathrm{O}$ stars, the observed cores must obtain additional mass from outside their observationally derived boundaries. The observations suggest that the cores in this protocluster are being fed via global infall from the very massive reservoir $\left(\sim 1500 M_{\odot}\right)$ of gas within which the protocluster is embedded.

S.N.L. thanks Stella Offner, Phil Myers, David Wilner, Tyler Bourke, Rowan Smith, and Paul Ho for thoughtful comments, Roberto Galvan-Madrid for comments on a draft of the paper, and Mark Krumholz for a stimulating discussion on the analysis. S.N.L. gratefully acknowledges support of this research through funding as a Submillimeter Array Fellow. The Submillimeter Array is a joint project between the Smithsonian Astrophysical Observatory and the Academia Sinica Institute of Astronomy and Astrophysics and is funded by the Smithsonian Institution and the Academia Sinica. This research has made use of NASA's Astrophysics Data System.

\section{REFERENCES}

Becker, R. H., White, R. L., Helfand, D. J., \& Zoonematkermani, S. 1994, ApJS, 91, 347

Benjamin, R. A., et al. 2003, PASP, 115, 953

Beuther, H., Schilke, P., Sridharan, T. K., Menten, K. M., Walmsley, C. M., \& Wyrowski, F. 2002, A\&A, 383, 892

Beuther, H., Walsh, A. J., \& Longmore, S. N. 2009, ApJS, 184, 366

Bica, E., Dutra, C. M., Soares, J., \& Barbuy, B. 2003, A\&A, 404, 223

Carey, S. J., et al. 2009, PASP, 121, 76

Carolan, P. B., et al. 2009, MNRAS, 400, 78

Caswell, J. L. 1998, MNRAS, 297, 215

Cesaroni, R. 2005, in IAU Symp. 227, Massive Star Birth: A Crossroads of Astrophysics, ed. R. Cesaroni, M. Felli, E. Churchwell, \& M. Walmsley (Cambridge: Cambridge Univ. Press), 59

Chen, H., Welch, W. J., Wilner, D. J., \& Sutton, E. C. 2006, ApJ, 639, 975

Cox, A. N. 2000, Allen's Astrophysical Quantities (New York: Springer)

Cyganowski, C. J., Brogan, C. L., Hunter, T. R., \& Churchwell, E. 2009, ApJ, 702,1615

De Buizer, J. M., Redman, R. O., Longmore, S. N., Caswell, J., \& Feldman, P. A. 2009, A\&A, 493, 127

de Wit, W. J., Hoare, M. G., Oudmaijer, R. D., \& Lumsden, S. L. 2010, A\&A, 515,45

Elmegreen, B. G. 1989, ApJ, 338, 178

Fallscheer, C., Beuther, H., Zhang, Q., Keto, E., \& Sridharan, T. K. 2009, A\&A, 504, 127

Fomalont, E. B. 1999, in ASP Conf. Ser. 180, Synthesis Imaging in Radio Astronomy II, ed. G. B. Taylor, C. L. Carilli, \& R. A. Perley (San Francisco, CA: ASP), 301

Forster, J. R., \& Caswell, J. L. 1989, A\&A, 213, 339

Froebrich, D. 2005, ApJS, 156, 169

Galván-Madrid, R., Keto, E., Zhang, Q., Kurtz, S., Rodríguez, L. F., \& Ho, P. T. P. 2009, ApJ, 706, 1036

Garay, G., \& Lizano, S. 1999, PASP, 111, 1049

Girart, J. M., Beltrán, M. T., Zhang, Q., Rao, R., \& Estalella, R. 2009, Science, 324, 1408

Green, S. 1986, ApJ, 309, 331

Harju, J., Lehtinen, K., Booth, R. S., \& Zinchenko, I. 1998, A\&AS, 132, 211

Hatchell, J., Thompson, M. A., Millar, T. J., \& MacDonald, G. H. 1998, A\&AS, 133,29

Hill, T., Burton, M. G., Minier, V., Thompson, M. A., Walsh, A. J., HuntCunningham, M., \& Garay, G. 2005, MNRAS, 363, 405 
Hill, T., Longmore, S. N., Pinte, C., Cunningham, M. R., Burton, M. G., \& Minier, V. 2010, MNRAS, 402, 2682

Hill, T., Thompson, M. A., Burton, M. G., Walsh, A. J., Minier, V., Cunningham, M. R., \& Pierce-Price, D. 2006, MNRAS, 368, 1223

Hofner, P., \& Churchwell, E. 1996, A\&AS, 120, 283

Ho, P. T. P., Moran, J. M., \& Lo, K. Y. 2004, ApJ, 616, L1

Kauffmann, J., Bertoldi, F., Bourke, T. L., Evans, N. J., II., \& Lee, C. W. 2008, A\&A, 487, 993

Keto, E. R. 1990, ApJ, 355, 190

Keto, E. 2003, ApJ, 599, 1196

Keto, E. 2007, ApJ, 666, 976

Keto, E., \& Klaassen, P. 2008, ApJ, 678, L109

Keto, E., Rybicki, G. B., Bergin, E. A., \& Plume, R. 2004, ApJ, 613, 355

Keto, E., \& Zhang, Q. 2010, MNRAS, 406, 102

Kruegel, E., \& Siebenmorgen, R. 1994, A\&A, 288, 929

Krumholz, M. R., Klein, R. I., \& McKee, C. F. 2007, ApJ, 656, 959

Krumholz, M. R., Klein, R. I., McKee, C. F., Offner, S. S. R., \& Cunningham, A. J. 2009, Science, 323, 754

Krumholz, M. R., \& McKee, C. F. 2008, Nature, 451, 1082

Lada, C. J., \& Lada, E. A. 2003, ARA\&A, 41, 57

Lamers, H. J. G. L. M., \& Cassinelli, J. P. 1999, Introduction to Stellar Winds (Cambridge: Cambridge Univ. Press)

Leurini, S., Beuther, H., Schilke, P., Wyrowski, F., Zhang, Q., \& Menten, K. M. 2007, A\&A, 475, 925

Longmore, S. N., \& Burton, M. G. 2009, PASA, 26, 439

Longmore, S. N., Burton, M. G., Barnes, P. J., Wong, T., Purcell, C. R., \& Ott, J. 2007, MNRAS, 379, 535

Longmore, S. N., Burton, M. G., Keto, E., Kurtz, S., \& Walsh, A. J. 2009, MNRAS, 399, 861

Longmore, S. N., Burton, M. G., Minier, V., \& Walsh, A. J. 2006, MNRAS, 369,1196

Lovas, F. J. 2004, J. Phys. Chem. Ref. Data, 33, 177

Mathis, J. S., Rumpl, W., \& Nordsieck, K. H. 1977, ApJ, 217, 425

Offner, S. S. R., Klein, R. I., McKee, C. F., \& Krumholz, M. R. 2009, ApJ, 703, 131

Peretto, N., André, P., \& Belloche, A. 2006, A\&A, 445, 979

Peretto, N., Hennebelle, P., \& André, P. 2007, A\&A, 464, 983

Peters, T., Banerjee, R., Klessen, R. S., Mac Low, M., Galván-Madrid, R., \& Keto, E. R. 2010, ApJ, 711, 1017

Pillai, T., Wyrowski, F., Carey, S. J., \& Menten, K. M. 2006, A\&A, 450, 569

Pillai, T., Wyrowski, F., Hatchell, J., Gibb, A. G., \& Thompson, M. A. 2007, A\&A, 467, 207

Purcell, C. R., Longmore, S. N., Burton, M. G., Walsh, A. J., Minier, V., Cunningham, M. R., \& Balasubramanyam, R. 2009, MNRAS, 394, 323

Purcell, C. R., et al. 2006, MNRAS, 367, 553
Qiu, K., Zhang, Q., Wu, J., \& Chen, H. 2009, ApJ, 696, 66

Ragan, S. E., Bergin, E. A., Plume, R., Gibson, D. L., Wilner, D. J., O’Brien, S., \& Hails, E. 2006, ApJS, 166, 567

Rathborne, J. M., Jackson, J. M., Chambers, E. T., Stojimirovic, I., Simon, R., Shipman, R., \& Frieswijk, W. 2010, ApJ, 715, 310

Rathborne, J. M., Jackson, J. M., \& Simon, R. 2006, ApJ, 641, 389

Rohlfs, K., \& Wilson, T. L. 2004, Tools of Radio Astronomy (4th rev. and English ed.; Berlin: Springer)

Scoville, N. Z., \& Kwan, J. 1976, ApJ, 206, 718

Scoville, N. Z., Sargent, A. I., Sanders, D. B., Claussen, M. J., Masson, C. R., Lo, K. Y., \& Phillips, T. G. 1986, ApJ, 303, 416

Shepherd, D. S., \& Churchwell, E. 1996, ApJ, 472, 225

Smith, R. J., Longmore, S., \& Bonnell, I. 2009, MNRAS, 400, 1775

Stahler, S. W., \& Palla, F. 2005, in The Formation of Stars, ed. S. W. Stahler \& F. Palla (Hoboken: Wiley-VCH), 865

Swift, J. J. 2009, ApJ, 705, 1456

Takahashi, S., Ho, P. T. P., Tang, Y., Kawabe, R., \& Saito, M. 2009, ApJ, 704 1459

Tang, Y., Ho, P. T. P., Girart, J. M., Rao, R., Koch, P., \& Lai, S. 2009a, ApJ, 695, 1399

Tang, Y., Ho, P. T. P., Koch, P. M., Girart, J. M., Lai, S., \& Rao, R. 2009b, ApJ, 700,251

Tang, Y., Ho, P. T. P., Koch, P. M., \& Rao, R. 2010, ApJ, 717, 1262

Thompson, M. A., Hatchell, J., Walsh, A. J., MacDonald, G. H., \& Millar, T. J. 2006, A\&A, 453, 1003

Val'tts, I. E., Ellingsen, S. P., Slysh, V. I., Kalenskii, S. V., Otrupcek, R., \& Larionov, G. M. 2000, MNRAS, 317, 315

Walsh, A. J., Bourke, T. L., \& Myers, P. C. 2006, ApJ, 637, 860

Walsh, A. J., Burton, M. G., Hyland, A. R., \& Robinson, G. 1998, MNRAS, 301,640

Walsh, A. J., Lo, N., Burton, M. G., White, G. L., Purcell, C. R., Longmore, S. N., Phillips, C. J., \& Brooks, K. J. 2008, PASA, 25, 105

Walsh, A. J., Myers, P. C., \& Burton, M. G. 2004, ApJ, 614, 194

Wilner, D. J., Wright, M. C. H., \& Plambeck, R. L. 1994, ApJ, 422, 642

Wilson, T. L., \& Matteucci, F. 1992, A\&AR, 4, 1

Wood, D. O. S., \& Churchwell, E. 1989, ApJS, 69, 831

Wu, J., \& Evans, N. J., II. 2003, ApJ, 592, L79

Zhang, Q., Ho, P. T. P., \& Ohashi, N. 1998, ApJ, 494, 636

Zhang, Q., Hunter, T. R., Beuther, H., Sridharan, T. K., Liu, S., Su, Y., Chen, H., \& Chen, Y. 2007a, ApJ, 658, 1152

Zhang, Q., Hunter, T. R., Brand, J., Sridharan, T. K., Cesaroni, R., Molinari, S., Wang, J., \& Kramer, M. 2005, ApJ, 625, 864

Zhang, Q., Hunter, T. R., Brand, J., Sridharan, T. K., Molinari, S., Kramer, M. A., \& Cesaroni, R. 2001, ApJ, 552, L167

Zhang, Q., Sridharan, T. K., Hunter, T. R., Chen, Y., Beuther, H., \& Wyrowski, F. 2007b, A\&A, 470, 269

Zhang, Q., Wang, Y., Pillai, T., \& Rathborne, J. 2009, ApJ, 696, 268 